\title{
Exemples de recadrage du discours direct en français Pour une approche historique du discours théâtral
}

\author{
Cigada, Sara \\ Università Cattolica del Sacro Cuore (Milan, Italie) \\ sara.cigada@unicatt.it
}

\section{Introduction}

Pour cet article, nous avons voulu reprendre une suggestion proposée par Bernard Combettes lors du CMLF de 2012. Il avait souligné que les structures linguistiques changent de format et de fonction dans le temps. Ce phénomène est facilement appréciable, par exemple, pour ce qui concerne le lexique: un mot change de forme et de sens, sous l'influence du changement phonétique, de phénomènes systémiques déclenchés par l'interférence d'autres langues (emprunts qui «occupent » p. ex. un espace sémantique en modifiant par conséquent la valeur des mots qui font partie de cet espace), de l'usage et de besoins communicatifs liés au contexte historique, politique etc. Étudier l'histoire d'un lexème suppose donc la considération de chacun de ces niveaux, et d'autres, selon les cas. Combettes soulignait que ce même procédé devrait être appliqué à d'autres structures linguistiques, textuelles et, ajoutons-nous, discursives. Il parlait par exemple de la structure et de la fonction de la description : si nous considérons la description des vêtements d'Énide habillée par Guenièvre dans le roman de Chrétien de Troyes, nous remarquons que le temps de la narration se prolonge d'une manière qui, aujourd'hui, apparaît peu naturelle, excessive. Si ces passages sont linguistiquement précieux, comme source documentaire sur les habits de l'époque, ils produisent aussi sur nous un effet d'extranéité presque physique : le texte s'avère aujourd'hui peu " proportionné ». On pourrait dire la même chose à propos des dialogues de la Princesse de Clèves, dans lesquels les personnages dissertent de leurs sentiments réciproques (Cigada 2005 : 212-213). Pour s'emparer des présupposés communicatifs permettant d'interpréter ces longues répliques, il ne suffit pas de rentrer dans le monde du récit : celui-ci est en effet clair, tout comme les sentiments et les relations qu'il inclut. Du point de vue du contenu nous percevons, il est vrai, une distance, par exemple entre la fidélité inconditionnelle de la jeune princesse de Clèves à son mari, vivant puis défunt, contre son sentiment même, et la pratique actuelle de la fidélité dans le mariage. Mais nous comblons cette distance - de manière un peu hâtive et superficielle peut-être - par des clichés historico-culturels. Ce qui nous demeure vraiment étranger, par contre, est la manière dont les personnages parlent, en termes de quantité, à cause de la longueur des répliques rapportées et des nombreux détails qu'elles contiennent. L'effet de distance relève autant (ou plus) de ce niveau, que de celui du contenu. À ce propos, deux implications de la suggestion de Combettes nous ont frappée.

1. D'abord, son idée d'étendre une méthodologie de recherche bien connue, en l'appliquant à des niveaux de la construction du texte (ou du discours) moins étudiés sous cette perspective. Parcourir systématiquement ce chemin pourrait donner comme résultat quelque chose de semblable à la compétence qu'un linguiste exploite pour l'analyse d'un lexème : il a une multitude de remarques à faire - et de questions à poser - devant n'importe quel mot, car il a une connaissance systématique des phénomènes divers qui peuvent l'affecter dans son évolution historique. Il domine en effet une méthodologie (ou une pluralité de méthodologies) d'analyse lexicale, permettant de questionner un mot de manière pertinente. La perspective de développer une compétence analogue concernant d'autres niveaux de la construction du discours a sans aucun doute de l'attrait.

2. Le deuxième aspect concerne la langue française, l'objectif étant d'étudier l'identité «française » des phénomènes linguistiques analysés. En effet, la démarche préconisée par Combettes épouse cette perspective, car la comparaison entre les différents «états de langue » permettrait de mieux connaître la langue française en soi, mais elle permettrait aussi d'y discerner des états linguistiquement et 
culturellement différents, tout en demeurant dans les limites de la même langue-culture française. Nous nous proposons en effet d'analyser le discours direct en tant que structure discursive se manifestant dans des «cultures » éloignées, en diachronie plutôt qu'en synchronie. Nous dressons donc l'hypothèse qu'il est possible de reconnaître des « états de culture » correspondant à des « états de langue ».

\section{Objet linguistique de la recherche}

Comme objet de cette recherche, nous avons choisi le discours rapporté (dorénavant DR) direct dans un contexte particulier, celui du discours de l'action dramatique dans le théâtre français. Attention : nous ne songeons pas du tout au théâtre comme «texte en discours direct ». Notre objet concerne le fait qu'un personnage rapporte la parole d'un autre, en le citant en discours direct (dorénavant DD). Un cas exemplaire : dans Phèdre, la mort d'Hippolyte ne se déroule pas sur scène; elle est racontée à son père, Thésée, par Théramène ( $\$ 4.5$ de cet article), qui insère dans son récit les derniers mots prononcés par le jeune prince mourant, en DD : après avoir rapporté les derniers mots d'Hippolyte, Théramène reprend la parole pour terminer son récit. Racine utilise donc le DD comme l'un des éléments de ce récit.

Nous avons choisi le théâtre à cause de la pertinence, qualitative et quantitative, de ce genre dans l'histoire de la culture française. Il est relativement facile d'établir un (ou plusieurs) corpus, par auteur, par époque, par typologie... et donc d'utiliser ces textes de manière méthodologiquement correcte pour une recherche, même un peu "quantitative-mécanique», au départ du moins. Nous nous référons, par exemple, à la possibilité de chercher de manière automatique les guillemets qui ouvrent les passages en DD en utilisant des éditions numériques des ouvrages, même si cela n'est pas toujours suffisant, comme nous le verrons plus loin. En outre, l'interdiscours du théâtre est extrêmement riche : il suffit de penser aux textes des auteurs qui commentent ou expliquent leurs pièces, à la critique, à l'histoire des mises en scène, aux déclarations d'acteurs fameux qui ont joué ces rôles..., mais aussi aux rapports explicites entre théâtre et éducation, théâtre et idéologie, théâtre et pouvoir...

\subsection{Le DD dans le discours théâtral}

Du point de vue de sa nature discursive, le DD enchâssé dans le discours théâtral exalte la nature dramatique que Bakhtine attribue même «à l'énoncé le plus simple» (Todorov 1981: 75-76). Si le discours théâtral est conçu - dès sa formulation - pour réaliser une action (drame), la mise en scène du DD dans le drame multiplie les effets discursifs. En outre, la récitation pose à l'acteur le problème de mettre en scène la voix qu'il cite. Cet aspect est même inscrit dans ce type de DD et il serait très intéressant de réaliser un travail d'analyse du discours pour comparer les différentes performances de grands acteurs dont nous possédons les enregistrements, pour étudier les manières qu'ils ont inventées pour réaliser ce type d'action ainsi que les discours concernant ce sujet. En même temps, l'étude de la voix au sens physique du mot nous rapprocherait de manière très pertinente de ce que Bakhtine évoque en parlant de l'intonation (Todorov 1981: 194 ss ; 301 ss et passim). Nous ne voulons pas, pour autant, borner notre regard à l'organisation polyphonique de ces discours, au sens bakhtinien de "discours littéraire» (cf. Nowakowska 2005: 23-24 et passim), mais surtout prendre en compte leur nature dialogique.

Étudier la dialogisation dans le contexte du discours théâtral pose un problème, parce que (selon la caractérisation fournie par Bres $2005: 55$ ) le discours-pièce manifeste une nature monologale (en tant qu'il est créé par un seul auteur). Nous en considérons toutefois le dialogisme (en tant que discours s'adressant à un auditoire dont la «culture » - y compris la langue - est commune à l'auteur et prise en charge par celui-ci). La question est justement là : si la manière d'organiser le discours (comme par exemple la manière d'insérer les DD ou les autres paramètres que nous allons étudier) change dans le temps, il faut pouvoir saisir et «mesurer » ce changement. Ce changement concerne le système langueculture, qui appartient en commun aux interlocuteurs (dans notre cas, à l'auteur de la pièce et à son auditoire). Or, la «manière d'organiser le dialogisme » constitue un phénomène beaucoup plus vaste que l'insertion d'un DD dans la réplique d'une pièce. Toutefois, justement du fait que le DR peut être 
considéré comme « la partie émergée de l'iceberg dialogisme 》 (Bres 2005 : 59), nous avons expérimenté cette voie pour sonder notre hypothèse.

Du point de vue linguistique, en effet, l'objet DD est «plus facile » à saisir, car il a une forme linguistique (dont la manifestation est morphosyntaxique, mais aussi typographique) assez déterminée - encore plus que d'autres types de discours rapportés donc - ce qui donne un ancrage solide à la recherche pour ce qui concerne l'individuation des données pertinentes. En même temps, ce type de DD présente un rapport immédiat soit avec le dialogisme, soit avec la polyphonie, éléments centraux de l'organisation du discours. En effet, la stratégie discursive en DD que nous souhaitons étudier se trouve au carrefour entre polyphonie et dialogisme dans les sens identifiés par Rosier $(2008: 38-40)$ : il y a, dans ces occurrences du DD, du dialogisme, à savoir « la capacité de l'énoncé à faire entendre, outre la voix de l'énonciateur, une (ou plusieurs) autre(s) voix qui se hiérarchise(nt) du point de vue énonciatif», mais aussi de la polyphonie car l'effet de «mise en scène des différents points de vue et voix dans le discours » est, si l'on peut dire, réalisé au pied de la lettre. Sur dialogisme vs polyphonie nous renvoyons aussi à Bres - Mellet (2009 : 6-9) : dans le contexte du discours que nous étudions, la « conception théâtrale de l'énonciation » n'est donc pas prise au sens métaphorique.

Cependant, il nous semble évident qu'il ne serait pas trop raisonnable de nous proposer d'ores et déjà un résultat théorique, du fait qu'il faut tout d'abord vérifier le côté empirique : nous exploitons donc les catégories issues des travaux cités, de manière cohérente, pour vérifier si les données linguistiques subsistent. Pour ce qui concerne le "cadre énonciatif », nous considérons que, grâce au DD, les répliques des énonciateurs-personnages (ces répliques constituant le discours-pièce) enchâssent dans l'énonciation principale d'autres énonciateurs (Rosier 2008: 40 et passim) et leurs énoncés. On remarque que, par conséquent, l'organisation énonciative change selon les rapports qui relient les énonciateurs entre eux, aux différents niveaux d'enchâssement. Les 'recadrages' énonciatifs dépendent soit de la structure de l'enchâssement, soit de la nature énonciative du DR (modalité et temps du verbe citant). L'intérêt de cette analyse dépend tout d'abord de l'organisation de l'enchâssement, parce que les énonciateurs cités en DD peuvent coïncider ou non avec les personnages.

Pour ce qui concerne l'auteur et l'auditoire/lecteur (c'est-à-dire le producteur et le destinataire physiques du discours théâtral), nous en considérons l'existence comme un présupposé, ainsi que le caractère monogal de la pièce, la nature fictive des dialogues, du 'recadrage' et du caractère dialogal du discourspièce (cf. § 2.1): les énonciateurs et les énoncés "enchâssés » ne représentent en effet que des «feuilletages» de l'auteur et du discours-pièce ${ }^{1}$. Finalement, nous avons adopté une terminologie qui permet de repérer le plus immédiatement possible le cadre énonciatif: énonciateur-personnage et allocutaire-personnage ; énoncé ou discours enchâssant ; énonciateur et allocutaire du discours enchâssé, etc. Tous ces phénomènes ne relèvent que du dialogisme.

\subsection{Repères bibliographiques}

À propos du DD nous soulignons tout d'abord sa nature «intermédiaire » - en tant que linguistique entre structure et fonction : Rigotti - Rocci (2006), ce qui justifie sa présence dans des discours très variés, un certain dégré de souplesse de sa structure et sa versatilité par rapport aux fonctions qu'il peut remplir (cf. Cigada 2012a). Sur la structure linguistique du DD en tant que forme particulière du DR, cf. Rosier (2008). À propos de l'autonymie comme caractéristique du DD, cf. la bibliographie citée dans Cigada (2012a). La distinction entre «autonymie de langue » et «autonymie de parole» se montre intéressante par rapport au corpus étudié dans cet article, surtout pour comparer quelques uns des DD, plus évidemment «autonymiques», avec d'autres qui le paraissent moins. Nous n'allons toutefois pas parcourir ce chemin.

Il faut dire aussi que le phénomène spécifique que nous avons abordé ne semble pas avoir été traité de manière systématique auparavant: cf Siouffi $(2013: 141)$ sur le Cid, ou Dufiet (2005) qui parle de «prouesse discursive » à propos de Molière, ou encore Petitjean (2005) sur Marivaux. Dans le joli volume Le discours rapporté dans tous ses états édité par Lopez Muños, Marnette et Rosier (2004), ce type de DD n'est pas cité. La reprise en écho revient dans Granier (2003 : 217-231) et Salvan (2005) : par 
ailleurs, Sorin Stati affrontait déjà le problème (1991: 56-58), à propos de la «fonction écho », avec un exemple tiré de Cocteau. Authier-Revuz (2003 : 86-87) considère la scène du Misanthrope qui se base sur l'autocitation.

Pour ce qui concerne les fonctions de ce type de DD, Adam (1992:188-192) ne considère pas l'insertion d'un DD dans le récit de Théramène, quand il analyse la mort d'Hippolyte. Il considère par contre 'Le récit dans la conversation' (Adam 1992 : 172-175). Adam considère aussi le dialogue entre Agnès et le jeune homme rapporté par Agnès à Arnolphe en DD (Adam 1992: 172-175). L'auteur n'étudie toutefois que l'organisation interne du récit, et plus particulièrement la fonction des questions et des commentaires d'Arnolphe, qui orientent la construction de la narration d'Agnès : de fait, Adam réduit le DD à un élément quelconque du récit d'Agnès. Comme l'affirme Calaseru en clôture de son livre Testuali parole (2004 : 211), «Il discorso riportato ci appare come un punto di osservazione strategico anche per il discorso in genere» (cependant, Calaresu ne considère pas le type d'objet dont nous nous occupons ici) ${ }^{2}$.

\section{$3 \quad$ But, méthode et plan de l'article}

Ce que nous nous proposons donc ici est d'émettre une première hypothèse pour vérifier si la suggestion de Combettes donne un résultat intéressant et, d'autre part, de mettre à nouveau en cause la structure linguistique du DD et les fonctions qu'elle peut remplir, dans le contexte d'un discours précis, celui de l'action théâtrale. Le but de cet article est partant de sonder le terrain, pour donner un aperçu général de la faisabilité d'une telle recherche.

Nous avons commencé ce sondage avec beaucoup de liberté par l'analyse de sept pièces, sans tenir compte du genre auquel elles appartiennent. En voici la liste et la date de leur création : 1637 le Cid (Pierre Corneille); 1666 le Misanthrope (Molière) ; 1677 Phèdre (Jean Racine) ; 1725 l'Âle des esclaves (Marivaux) ; 1897 Cyrano de Bergerac (Edmond Rostand) ; 1928 Topaze (Marcel Pagnol); 1953 En attendant Godot (Samuel Beckett). Mais, après une longue réflexion, nous avons résolu de présenter les exemples suivant l'ordre diachronique renversé, du plus récent au plus ancien. La démarche est discutable, mais méditée: cette disposition permet de percevoir plus aisément, à notre avis, les différences entre les dialogismes.

Dans chacune de ces pièces, nous avons pris en compte la totalité des DD. Si quelques exemples n'ont pas été analysés, nous l'avons signalé. La recherche automatique n'est en fait pas possible, car le DD, tout en étant reconnaissable grâce à sa structure syntaxique et discursive, n'est pas toujours marqué du point de vue typographique par des guillemets. Le discours rapporté en DD peut être oral ou bien écrit. Pour l'analyse, nous avons considéré la fréquence et la distribution des DD dans la pièce, les aspects morphologiques, le cadre énonciatif et ses variations/ recadrages et les aspects pragmatique-fonctionnels, selon une grille en quatre points que nous proposons ci-dessous.

1. Fréquence des DD et leur distribution. Les DD se trouvent distribués de manière inégale tout au long des pièces et d'une pièce à l'autre : ces données, concernant la fréquence des DD dans chaque pièce et leur collocation préférentielle, pourraient être pertinentes pour évaluer le changement de l'organisation discursive théâtrale au cours du temps. Nous avons partant mis en relief cet aspect, de manière descriptive.

2. Aspects morphosyntaxiques (concernant le cadre énonciatif et l'énoncé en DD). Modes et temps du verbe citant $^{3}$. À l'intérieur de l'énoncé en DD: termes d'adresse; formes de politesse et usage de tutoiement-vouvoiement ; exclamations/interjections ; phrases interrogatives, impératives, exclamatives.

3. Cadre énonciatif. Autour du DD, les répliques des énonciateurs-personnages (fictifs) enchâssent dans l'énonciation principale d'autres 'énonciateurs' (fictifs) et leurs énoncés. Nous avons étudié les relations entre les énonciateurs aux différents niveaux d'enchâssement (identité ou échange de rôle énonciatif entre personnages, énoncés exogènes). Le dialogisme en est élaboré de manière différente dans les différentes pièces. 
4. Aspects pragmatique-fonctionnels. Réinterprétation pragmatique du DD rapporté et amalgames pragmatiques (selon l'expression de Kerbrat-Orecchioni) ; exploitation du cadre pour des effets de sens ; éthopée; narrativisation; argumentation; implication émotive. Les remarques sur la manipulation rhétorique que l'on trouvera au cours de l'analyse ont affaire à ce niveau de la description du discours : en effet, la rupture morphosyntaxique et le recadrage énonciatif entrainent des effets de sens spécifiques. Un énoncé en DD peut servir par exemple d'argument, mais il peut remplir d'autres fonctions (faire poursuivre la narration, montrer le caractère d'un personnage, etc.).

\section{Analyse des exemples}

\subsection{Godot}

Dans En attendant Godot, pièce en deux actes, le DD n'apparaît que dans le deuxième acte, où il est utilisé deux fois. Deux autres usages, toujours dans le deuxième acte, ont un statut ambigu, comme dans l'exemple qui suit: au début du deuxième acte, Vladimir chantonne à plusieurs reprises cette chansonnette.

\subsubsection{Exemple - Un chien vint dans l'office (Acte II, pp. 79-80 ${ }^{4}$ )}

Vladimir : Un chien vint dans l'office

Et prit une andouillette.

Alors à coups de louche

Le chef le mit en miettes.

Les autres chiens ce voyant

Vite vite l'ensevelirent

Au pied d'une croix en bois blanc

Où le passant pouvait lire :

Un chien vint dans l'office...

Il n'y a pas de verbe citant. L'énonciateur-personnage est seul sur scène, il n'y a pas d'allocutaire. L'énonciateur-cité a un statut indéfini, que l'on ne peut reconstruire que par la référence à plusieurs éléments de nature culturelle et contextuelle (voir plus bas). L'énoncé enchâssé ne s'adresse à personne. Le sujet de l'énoncé cité n'a aucun rapport avec les énonciateurs, à aucun niveau du discours. L'énoncé cité comporte un enchâssement secondaire : des énonciateurs (les autres chiens) et un allocutaire (le passant). L'énoncé cité est constitué par le texte écrit sur la croix en bois blanc, qui répète l'énoncé enchâssant. Virtuellement, cette structure se répète à l'infini. On pourrait considérer la mélodie comme marquant le statut discursif différent.

Du point de vue fonctionnel, ce discours commence comme un récit d'évènements, mais se poursuit comme un récit de paroles (écrites en épitaphe sur le tombeau du chien) : ce DR, à l'oral, se répète à l'infini grâce à sa structure de cité-et-citant ou de mise en abyme. La mélodie de la chanson, que Beckett aurait traduite de l'allemand au français (et ensuite en anglais), est reprise du Carnaval de Venise straussien $^{5}$ (cf. Sebellin 2003: 41-58). La superposition de plusieurs éléments, dont quelques-uns oxymoriques (mélodie fameuse, écrite en souvenir du violoniste feu Heinrich W. Ernst, ami de Strauss ; mélodie connue sous le titre de 'Carnaval' de Venise; mélodie fantaisiste et légère, bâtie sur la variation sur un thème très simple et employée pour une chanson enfantine ; contenu violent de l'histoire racontée et référence à la mort ; construction répétitive circulaire à l'infini ; traduction des paroles de l'allemand en français), fait peut-être de ce DD (s'il l'est) la clef de voûte de la pièce, du point de vue de l'exploitation discursive du dialogisme et de la polyphonie. Nous proposons en effet de considérer cette chanson comme du DR en DD, même si ce statut n'est pas indiscutable à cause de la nature autonymique du discours cité. Vladimir répète un discours dont on ne connaît pas le premier énonciateur, un discours d'enfant, de tous et de personne ${ }^{6}$. 


\title{
4.1.2 Exemple - Dis, Pense, cochon! (Acte II, p. 103)
}

\author{
Vladimir : Dis-moi de penser. \\ Estragon : Comment ? \\ V. : Dis, Pense, cochon! \\ E. : Pense, cochon! \\ V. : Je ne peux pas !
}

Ici l'acte de parole est ordonné en DD et ensuite exécuté. Le discours citant contient en effet un impératif (dis) qui exprime un ordre. Marque typographique : Pense, avec majuscule. Terme d'adresse : cochon. Du point de vue de l'organisation énonciative, l'énonciateur-personnage (Vladimir) s'adresse à l'allocutairepersonnage (Estragon) en lui demandant d'exécuter un énoncé en DD dont l'allocutaire (Estragon) sera l'énonciateur (Estragon), et l'énonciateur (Vladimir) sera l'allocutaire (Vladimir. Estragon dira donc a Vladimir "Pense, cochon !). Un enchâssement de la sorte présente une structure assez inusitée, sauf, peut-être, dans les jeux d'enfants : la reprise du DD précède-t-elle le DR même ? Le dialogisme en est-il déformé ? Remarquons par ailleurs que le DD ordonné exprime lui aussi un ordre, manifesté à l'impératif (Pense!).

Toute cette scène pourrait être considérée du point de vue discursif comme la reprise de la rencontre avec Pozzo et Lucky du premier acte, du fait qu'elle est introduite par la proposition de Vladimir « On pourrait jouer à Pozzo et Lucky » (p. 102).

\subsubsection{Exemple - Memoria praeteritorum bonorum (Acte II, p. 121)}

Vladimir : [...] laisse-le tranquille. Ne vois-tu pas qu'il est en train de se rappeler son bonheur. (Un temps.) Memoria praeteritorum bonorum - ça doit être pénible.

Il n'y a pas de verbe introducteur, mais la citation est marquée soit typographiquement, par l'italique, soit prosodiquement, par la pause indiquée dans la glose. Toutefois, comme pour 4.1.1, le statut de cette citation est ambigu. L'énonciateur reprend en variation (renversement sémantique) le dicton de Cicéron « etiam iucunda est memoria praeteritorum malorum » (« le souvenir des maux passés est agréable », Cic. de Finibus bonorum et malorum, II, 105, texte retrouvé sur Perseus, qui devient donc « le souvenir des biens passés est pénible »). Cicéron citerait lui-même un proverbe grec issu d'un vers d'Euripide. Même si l'énoncé n'est pas attribué, il est donc possible d'en reconstruire le premier énonciateur; en tant que doxa, toutefois, l'énoncé n'appartient à personne et s'adresse à tous.

La fonction de cet énoncé est finalement argumentative : il sert à justifier l'ordre que Vladimir donne à Estragon de ne pas déranger Pozzo.

\subsubsection{Exemple - Un autre me regarde, en se disant, II dort (Acte II, p. 128)}

Le DD n'est pas marqué typographiquement, mais la majuscule ainsi que la structure syntaxique l'indiquent de manière claire, comme dans 4.1.2. Le verbe introducteur est en disant, marqué par la réflexivité $(s e)$.

Vladimir : [...] Moi aussi, un autre me regarde, en se disant, Il dort, il ne sait pas, qu'il dorme. (Un temps.)

L'énonciateur (un autre) n'est pas précisé ; il s'adresse à lui-même. Dans cet exemple, l'effet discursif est curieux parce que l'énonciateur-personnage qui rapporte le DD (Vladimir) est censé dormir, ce qui impliquerait qu'il n'a pas pu entendre le discours de « l'autre ». En fait il ne dort pas mais parle.

On pourrait dire que ce dialogisme est fonctionnel à l'absurde, parce que l'absurde relève de l'emploi insensé du cadre énonciatif et non pas du contenu du $\mathrm{DD}^{7}$. La rupture du dialogisme rompt en effet la distinction entre la réalité et le rêve. 


\subsection{Topaze}

Dans la comédie Topaze, Marcel Pagnol insère plusieurs DR directs et aussi des textes écrits qui sont lus sur la scène par Topaze (une lettre anonyme ; un article de journal et le document qui accompagne les Palmes). Les DD se trouvent en quantité majoritaire dans le troisième et le quatrième acte ${ }^{8}$. Dans les exemples 4.2.1, 4.2.2, 4.2.3 Topaze, hanté par les abus qu'il est en train de commettre, voit partout des accusateurs.

\subsubsection{Exemple - Tripoteur ! Tripoteur ! Tripoteur ! (Acte III, scène 2, p. 167)}

Topaze : [...] le bruit de leurs moteurs m'arrivait encore, et savez-vous ce qu'ils disaient, ces moteurs ? Ils disaient : «Tripoteur ! Tripoteur ! Tripoteur ! " Et les brosses obliques, en frôlant les pavés, chuchotaient: "Topaze escroc! Topaze escroc !».

Les verbes introducteurs à l'imparfait signalent une action répétée (disaient, chuchotaient). Topaze s'adresse à Suzy. Les énonciateurs dont le discours est rapporté sont les moteurs et les brosses des balayeuses, objets dont la pièce s'occupe largement mais qui n'ont jamais le statut d'énonciateurs en tant que personnages, si ce n'est dans ce DD, enchâssé dans une réplique de Topaze. Les énoncés en DD concernent Topaze lui-même, qui se considère comme l'objet et la cible de ce discours (qui n'existe pas).

Le DD sert donc, en fonction d'éthopée, pour animer ces énonciateurs fictifs, ce qui montre l'obsession de Topaze ${ }^{9}$.

\subsubsection{Exemple - Cachât ! (Acte III, scène 2, p. 167)}

En fonction typiquement autonymique, nous remarquons la reprise de la correction de la forme verbale dans l'exemple suivant, et plusieurs autres reprises en écho que nous ne considérons pas :

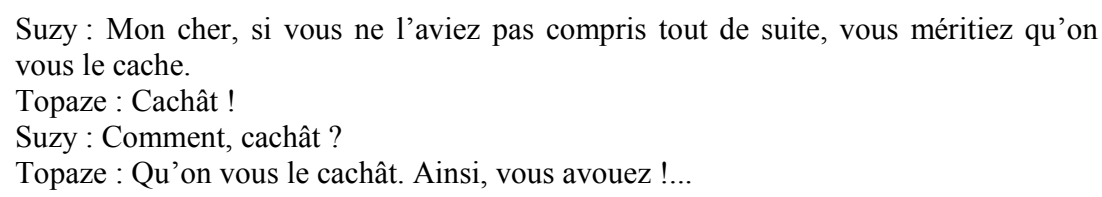

La reprise en écho ne prévoit pas dans ce cas-là de verbe introducteur. La phrase interrogative (Comment, cachât ?) pose cependant une question relative à l'énoncé (Cachât!) de Topaze, telle «Quel est le sens du propos que vous venez d'énoncer?».

\subsubsection{Exemple - le sympathique idiot (Acte III, scène 2, p. 167)}

Topaze : $[\ldots] \mathrm{j}$ 'entendis une conversation effroyable.

Suzy : Effroyable?

Topaze : Hideuse. Mais pleine de sens pour moi. M. Castel-Bénac disait : «Chérie, pourquoi as-tu invité le sympathique idiot?» et vous avez répondu: «Le sympathique idiot est très utile et il faut un peu l'amadouer $»$. Le sympathique idiot, c'était moi. Quant au mot «chérie », il m'a suffisamment renseigné sur la nature de vos relations avec cet homme.

L'imparfait disait du verbe citant constitue l'arrière-plan de l'action discursive, ponctuelle, véritablement visée, vous avez répondu. À remarquer la valeur, différente, de l'imparfait habituel (p. ex. Et je disais à Mme Muche: «S'il veut partir, je le laisserai libre!», p. 198; Elles me répondaient toujours: «Monsieur le directeur n'est pas là », p. 234) qui peut correspondre au présent habituel (on me répond: «Monsieur est chez son tailleur», p. 215). Encore différent, l'imparfait de Rostand (Le Duc : [...] Et quelqu'un me disait, hier, au jeu, chez la Reine :/ « Ce Cyrano pourrait mourir d'un accident », Acte V, scène 2, p. 292) qui, uni au pronom quelqu'un, opacifie la source (l'identité de l'énonciateur). Le DD reproduit le tutoiement entre Castel-Bénac et Suzy, tandis que Topaze continue de vouvoyer Suzy. 
Le dialogue rapporté en DD n'a jamais été joué sur scène : son contenu et ses conséquences sont appris par le spectateur au moment même où Topaze accuse Suzy d'avoir trompé sa confiance. Le cadre énonciatif comporte en effet Topaze et Suzy comme personnages, tandis que les deux répliques rapportées en DD par Topaze ont été respectivement prononcées par son patron (Castel-Bénac) et par sa maîtresse (Suzy). Les deux énonciateurs des énoncés enchâssés sont donc des personnages, doués de parole dans la pièce. Dans cet exemple, toutefois, le dialogue rapporté s'est déroulé entre deux énonciateurs (Suzy et Castel-Bénac) dont aucun ne coïncide avec l'énonciateur actuel (Topaze) : celui-ci l'a entendu de manière directe, mais hasardeuse, parce qu'il ne participait pas du cadre énonciatif du dialogue entre Suzy et Castel-Bénac. L'énonciateur-Topaze reconstruit donc à posteriori le cadre énonciatif de départ constitué par deux énonciateurs à plein statut plus un allocutaire à statut mineur (non intentionnel ni par les énonciateurs ni par lui-même). Le contexte exige une interprétation de littéralité presque totale du DR.

Il y a en outre la reprise en écho de sympathique idiot dans la conversation entre Castel-Bénac et Suzy et ensuite autonymique dans le discours de Topaze ; chérie est une reprise autonymique du discours de Castel-Bénac insérée dans celui de Topaze.

L'ensemble des informations reconstruites à partir de cette construction discursive a une fonction narrative, du fait qu'il permet de faire évoluer les rapports entre les personnages en constituant une nouvelle étape de la pièce.

\subsubsection{Exemple - ce que je dirais à mes élèves (Acte IV, scène 4, p. 239)}

Topaze : Sais-tu ce que je dirais à mes élèves? (Il s'adresse soudain à sa classe du premier acte.) «Mes enfants, ... »

La forme je dirais introduit un DD fictif, qui pourrait se dérouler dans le présent ou l'avenir. Bres Mellet (2009: 6 et passim) ont recours au verbe feuilleter, pour décrire le rapport multiple entre les énonciations. Si ce phénomène caractérise le discours théâtral en tant que « dialogue montré masquant un dialogisme interne, qui fait s'exprimer des voix hiérarchisées » (Salvan $2004: 276$ ), ce « feuilletage » est démasqué lors de l'enchâssement d'un énoncé en DD, surtout si l'énonciateur en est le même, comme dans cet exemple. Le verbe citant conditionnel (je dirais) met en relation le monde réel du discours-pièce (ce que Topaze a donc effectivement dit à ses élèves et qu'il est censé professer encore) avec le monde conditionnel du DD (ce qu'il dirait aujourd'hui s'il se trouvait à nouveau en classe et qu'il juge être le plus correct), les deux mondes étant reliés par le biais de l'énonciateur (Topaze). L'allocutaire Tamise, son ancien collègue à l'école, est témoin du changement du discours tenu par Topaze et donc du changement de l'énonciateur.

Du point de vue du récit, en effet, ce DD constitue la charnière entre l'ancien et le nouveau Topaze.

\subsection{Cyrano}

Cette pièce est très riche en $\mathrm{DD}$, ce qui est assez compréhensible si l'on songe que l'action tourne autour de la parole, authentique et/ou fictive, de Cyrano et Christian. Nous n'avons choisi que quelques exemples en en laissant beaucoup d'autres de côté ${ }^{10}$. La parole "prêtée » par Cyrano à Christian soustend en effet toute la pièce (Christian: Il me faudrait de l'éloquence ! Cyrano : Je t'en prête !/ Toi, du charme physique et vainqueur, prête-m' en/ Et faisons à nous deux un héros de roman!/ Christian : Quoi ? Cyrano : Te sentirais-tu de répéter les choses/ Que chaque jour je t'apprendrais ?... Acte II, scène 10, p. 155). Le dialogisme joue un rôle décisif dans la scène du balcon, dont l'effet émotif découle de l'identification retrouvée entre l'énonciateur (Cyrano) et l'auteur de la parole (Cyrano) : c'est en effet Cyrano qui, en se cachant dans la nuit et feignant d'être Christian, parle directement à Roxane sans qu'elle le reconnaisse (Acte III, scène 7-9, pp. 187-199). De plus, Cyrano récite souvent les lettres qu'il est en train d'écrire à Roxane ; Roxane déclame à Cyrano ces lettres dont Cyrano est le véritable auteur, en les croyant de Christian (Acte III, scène 1, p. 166-7). Finalement, l'anagnorèse est caractérisée par un dialogisme très fort: Cyrano lit à haute voix la dernière lettre qu'il a écrite à Roxane de la part de 
Christian, dont Roxane pense toujours qu'elle a été écrite par Christian; Roxane se rend compte en l'écoutant qu'il en connaît déjà les mots et, finalement, elle comprend que la voix de l'écrivain décédé quatorze ans auparavant, la voix écrite et lue, la voix du lecteur, sont la même : Cyrano se réapproprie ce qu'il est en train de lire et Roxane comprend qu'il l'aime et qu'il est maintenant en train de mourir (Cyrano : Et je crie : "Adieu !... ». Acte V, p. 305). Le feuilletage discursif est donc au cœur de la pièce.

\subsubsection{Exemple - On pouvait dire [...] vous m'auriez dit (Acte I, scène 4, pp. 72-74)}

Cyrano : [...] On pouvait dire... Oh ! Dieu !... bien des choses en somme/ En variant le ton, - par exemple, tenez:/ Agressif: «Moi, monsieur, si j’avais un tel nez/ Il faudrait sur-le-champ que je me l'amputasse !»/ Amical : « Mais il doit tremper dans votre tasse:/ Pour boire, faites-vous fabriquer un hanap $! » \ldots{ }^{11}$

Ce long passage, où le discours citant alterne avec le discours cité en en caractérisant le "ton », se conclut par un tiret qui correspond à celui qui ouvre la liste des exemples (cf. extrait ci-dessus). Après quoi Cyrano garde la parole pour terminer sa tirade :

Cyrano : $[\ldots]$ - Voilà ce qu'à peu près, mon cher, vous m'auriez dit/ Si vous aviez un peu de lettres et d'esprit/...

Rostand entoure ce DD par un discours citant qui le précède et un discours citant qui le suit, les deux discours citants ayant la même fonction. Toutefois, le passage de la forme impersonnelle (on pouvait dire) à la formule de politesse à la deuxième personne du pluriel (vous m'auriez dit) joue un rôle important car il permet de viser la cible des insultes qui vont suivre. Comme dans l'exemple 4.2.4, ces verbes introducteurs modalisent le rapport entre le discours-pièce et le DD, en reliant cette fois-ci une condition présente irréelle (si vous aviez...) à une action qui n'a pas eu lieu (vous m'auriez dit). Dans le discours qu'il suggère à son allocutaire (le vicomte), Cyrano se fait vouvoyer et traiter de monsieur.

Dans cet exemple le vicomte, c'est-à-dire l'énonciateur du DD qui est enchâssé dans la réplique de Cyrano, coïncide avec l'allocutaire de la réplique (le vicomte), tandis que l'allocutaire de ce DD enchâssé (Cyrano) est l'énonciateur du discours enchâssant (Cyrano). Tous les deux sont personnages de la pièce, ayant donc le statut d'énonciateurs : le DD ne fait que renverser la direction du discours (dans la réplique, Cyrano s'adresse au vicomte ; par le DD enchâssé dans la réplique, le vicomte s'adresse à Cyrano).

\subsubsection{Exemple - Qu'est-ce que c'est encore que cette égratignure ? (Acte II, scène 6, p.} 120)

L'énonciateur (Roxane) rappelle à l'allocutaire (Cyrano) la question qu'elle lui posait autrefois lorsqu'ils étaient enfants. En ce faisant, elle découvre que le contexte réactualise la question (Cyrano a à présent la main blessée) :

Roxane : [...] Alors, jouant à la maman,/ Je disais d'une voix qui tâchait d'être dure :/ (Elle lui prend la main) «Qu'est-ce que c'est encore que cette égratignure ?»/ (Elle s'arrête stupéfaite) Oh ! C'est trop fort ! Et celle-ci!

Le verbe citant (je disais) comporte l'auto-citation d'un discours tenu habituellement dans un passé très éloigné. Comme dans l'exemple 4.2.4, un des résultats en est le feuilletage discursif. Toutefois, ce feuilletage se conclut ici par la découverte que la situation de référence du DD se reproduit au présent de manière identique : le feuilletage en est réduit à une seule couche, ramenant le passé au présent discursif. Énonciateur-personnage (Roxane) et allocutaire-personnage (Cyrano) coïncident tous deux avec l'énonciateur (Roxane) et avec l'allocutaire (Cyrano) enchâssés. À propos du verbe citant, remarquons aussi un des peu d'exemples comportant une note caractérisant la voix du point de vue phonétique (d'une voix qui tâchait d'être dure).

Dans cet échange, la fonction de l'énoncé rapporté, au départ narrative et référée au passé, est réinterprétée comme actuellement performative : l'interrogation rapportée change de statut et le récit de parole se transforme en une question véritable. 


\subsubsection{Exemple - Passez, señorita ! (Acte IV, scène 4, p. 244)}

Le DD permet de citer la parole de l'interlocuteur étranger dans sa langue, comme les Espagnols que Roxane a rencontrés sur son chemin vers le camp français :

Roxane : [...] Alors je répondais : «Je vais voir mon amant. »/ - Aussitôt l'Espagnol à l'air le plus féroce/ [...] S'inclinait en disant: «Passez, señorita !»/ [...] J'ai dit : mon amant, oui... pardonne !/ Tu comprends, si j’avais dit : mon mari, personne/ Ne m'eût laissé passer...

Comme dans 4.3.2, le verbe introducteur à l'imparfait (je répondais) signale une action discursive réitérée dans le passé. De même pour le verbe citant la réponse, où, toutefois, l'imparfait se déplace sur le verbe décrivant le geste qui accompagne le discours (s'inclinait), tandis que le verbe introducteur se trouve au gérondif (en disant). Le cadre énonciatif du DD comporte un échange dialogal entre Roxane, qui est aussi l'énonciateur de la réplique enchâssante, et l'Espagnol (plusieurs Espagnols en fait, qui lui ont posé la question au fur et à mesure qu'elle avançait dans son voyage). L'Espagnol n'a pas le statut de personnage dans la pièce, sa parole n'y entre donc que par le discours de Roxane. L'usage du terme d'adresse en espagnol (señorita), ainsi que la longue description de l'attitude du « hidalgo », suggère une interprétation ironique.

Puis Roxane reprend un élément de son propre discours pour se justifier devant son époux de l'avoir traité d'amant : ce mot, repris de manière autonymique (et donc littérale), est introduit par le verbe $j$ 'ai dit et deux points, ce qui suggère plutôt une auto-citation. Ensuite, elle explique son choix en s'imaginant par l'absurde avoir dit la vérité. Ici, le verbe citant est (si) j'avais dit, exprimant une condition irréelle, suivi lui aussi des deux points et du DD mon mari. Le statut de cette occurrence est plus ambigu du fait que l'expression mon mari n'a jamais été prononcée avant, il ne s'agit donc pas d'une reprise autonymique.

\subsubsection{Exemple - C'était une Fâcheuse (Acte V, scène 5, p 298)}

Dans ce dialogue figé, Cyrano raconte s'être adressé à la mort qui venait le chercher et ce faisant, il la désigne comme une Fâcheuse. Roxane ne comprend en effet pas de qui il parle.

Cyrano : Cousine, c'était une/ Fâcheuse.

Roxane : Vous l'avez renvoyée?

Cyrano : Oui, j'ai dit :/ Excusez-moi, mais c'est aujourd'hui samedi,/ Jour où je dois me rendre en certaine demeure;/ Rien ne m'y fait manquer: repassez dans une heure !

Le verbe citant (j'ai dit) renvoie à une action discursive ponctuelle dans un passé qui est proche (cf. repassez dans une heure). Le feuilletage de l'énonciateur comporte Cyrano-personnage et Cyranoénonciateur de l'énoncé enchâssé en DD. L'allocutaire par contre change: dans la réplique Cyrano s'adresse à Roxane tandis que, dans le DR, il s'adresse à la Fâcheuse. Dans son DR, Cyrano parle, de manière indirecte, de Roxane. Comme l'Espagnol, la Fâcheuse n'a pas le statut de personnage dans la pièce; mais, à la différence de l'Espagnol, elle ne parle pas, même comme énonciateur d'un DR. Toutefois, le fait d'être représentée comme allocutaire du DD présuppose son statut d'interlocuteur au même niveau que les autres. La construction du cadre énonciatif sert donc ici à conférer à la Mort le statut de personnage.

Sous cette forme mitigée, Cyrano s'excuse d'être arrivé en retard en expliquant sans se faire comprendre par Roxane ce qui s'est passé. Le spectateur par contre, renseigné par les répliques précédentes, se rend compte du vrai sens. L'effet d'éthopée est bâti sur l'emploi de la personnification, mais encore plus sur la participation de la Fâcheuse à l'énonciation. Même si, dans le contexte de cette scène, Rostand exploite l'équivoque pour mitiger l'effet sur Roxane, dans la scène suivante - quand la vérité est désormais connue de tout le monde - la personnification continue de jouer un rôle pertinent, car c'est en se battant que Cyrano décède. L'auteur met en scène la métaphore du dernier combat entre le protagoniste et la Mort en le représentant comme un duel effectif, pendant lequel Cyrano ne cesse de s'adresser (à la deuxième personne) à son ennemie et à ses copains : le Mensonge, les Préjugés etc. Cet exemple exploite 
la distance entre le discours monologal constitué par l'énoncé-pièce, échangé entre l'auteur et le spectateur, et le discours dialogal mis en scène, comme dans l'exemple 4.2.3.

\subsection{L'Île des esclaves}

Dans l'T̂le des esclaves, comédie en un acte, les DD n'apparaissent que dans la scène 3, lors de la description du caractère d'Euphrosine, faite en sa présence, par Cléanthis, sa servante, interrogée par Trivelin. Le DD apparaît plusieurs fois. D'abord Cléanthis l'utilise pour ridiculiser son ancienne patronne. Basculant entre différents types de discours indirects, Cléanthis emploie aussi le DD ([...] la journée sera glorieuse : Qu'on m'habille ! Madame verra du monde...).

\subsubsection{Exemple - Comment vous portez-vous, Madame ? (scène 3, pp. 48)}

Si Euphrosine a le visage fatigué parce qu'elle a mal dormi, elle utilise, selon Cléanthis, son discours pour se justifier devant ses amies.

Cléanthis : [...] il y a remède à tout : vous allez voir. Comment vous portez-vous, Madame? Très mal, Madame : j'ai perdu le sommeil ; il a huit jours que je ne ferme l'œil ; je n'ose pas me montrer, je fais peur. Et cela veut dire : Messieurs, figurez-vous que ce n'est point moi, au moins; ne me regardez pas ; remettez à me voir ; ne me jugez pas aujourd'hui ; attendez que j'aie dormi. J'entendais tout cela, moi...

Il n'y a pas de verbe citant, le dialogue est mis en scène grâce à l'expression « vous allez voir », par une métaphore visuelle. L'énonciateur de l'énoncé enchâssant, Cléanthis, ne participe pas de l'énonciation enchâssée, rapporté en DD, si ce n'est comme allocutaire aléatoire (J'entendais tout cela, moi). Elle s'adresse à Trivelin, qui jugera de la culpabilité d'Euphrosine. Cette dernière écoute l'accusation de sa servante. Les énonciateurs des DR en DD sont Euphrosine, l'un des personnages, et ses amies, qui par contre ne sont présentes dans la pièce qu'à travers ce cadre énonciatif (comme l'Espagnol de l'exemple 4.3.3). L'une et les autres se vouvoient et s'appellent Madame, avec un effet de politesse maniérée.

La conversation citée en DD est suivie d'une paraphrase, qui la traduit en son vrai sens. Cette paraphrase est introduite par l'expression et cela veut dire, deux points et le terme d'adresse Messieurs. La paraphrase est construite en effet comme un autre DD, adressé d'Euphrosine à ses allocutaires à la deuxième personne du pluriel.

La comparaison entre le sens affiché et la réalité montre la fausseté du premier. La comparaison entre le sens affiché et le sens sous-entendu montre un désir démesuré de n'être jugée que favorablement, possédant donc une fonction d'éthopée. Le DD apparaît ensuite dans une scène galante entre Euphrosine et un cavalier: Cléanthis y montre la coquetterie d'Euphrosine, sa manière frivole de s'attacher les hommes.

\subsubsection{Exemple - Continuez, folâtre, continuez (scène 3, pp. 49)}

Cléanthis : [...] vous parliez d'une femme qu'il voyait souvent. Cette femme-là est aimable, disiez-vous ; elle a les yeux petits, mais très doux ; et là-dessus vous ouvriez les vôtres [...] Vous réussîtes pourtant, le cavalier s'y prit; il vous offrit son cœur. À moi ? lui dîtes-vous. Oui, Madame, à vous-même, à tout ce qu'il y a de plus aimable au monde. Continuez, folâtre, continuez, dîtes-vous, en ôtant vos gants sous prétexte de m'en demander d'autres $[\ldots]$

Dans sa tirade, constituée par une série de répliques, Cléanthis s'adresse tantôt à Trivelin tantôt à Euphrosine. Dans la première réplique en DD, elle parle directement à sa patronne en la vouvoyant par la forme disiez-vous, qui constitue le verbe introducteur du DD. L'imparfait indique une action d'une certaine durée située dans le passé. Le DD n'est pas marqué du point de vue typographique, si ce n'est par la ponctuation. Ce premier DR est adressé d'Euphrosine au cavalier, tandis que Cléanthis, à nouveau, a 
assisté à la scène en tant que servante. Le cavalier n'est pas un personnage de la pièce, il n'existe discursivement qu'en tant qu'allocutaire (puis énonciateur) d'un discours cité.

Le deuxième DD présente le même cadre énonciatif, sauf pour le verbe introducteur (lui dîtes-vous) qui désigne une action ponctuelle. Le troisième $\mathrm{DD}$ manifeste la réponse du cavalier, sans verbe citant. Ce DD contient le terme d'adresse Madame. Le quatrième est introduit à nouveau par la forme dites-vous, accompagnée par la description du geste accompagnant la parole (en ôtant vos gants...). Ce dernier DD, comportant l'introducteur dîtes-vous, contient le terme d'adresse folatre, qui, tout comme le précédent, n'est possible qu'en DR direct.

Les paroles du cavalier, dont l'apparence est sincère dans le discours de Cléanthis, mettent encore davantage en évidence le caractère rusé d'Euphrosine, qui joue à ne pas comprendre qu'il est sérieux quand elle le traite de folatre. Le DD permet ici à Trivelin de juger par lui-même de l'attitude d'Euproshine, en comparant ses contenances à ses mots, ses contenances et ses mots à ceux du cavalier. La parole d'Euphrosine, comparée à ses actions, en démontre la frivolité et l'insincérité : ces séquences en DD revêtent donc une fonction d'éthopée mais aussi, en amalgame pragmatique, narrative (elles permettent au récit de se développer) et argumentative (les paroles d'Euphrosine prouvent son délit).

\subsubsection{Exemple - Un jour qu'elle pouvait m'entendre (scène 3, pp. 49-50)}

Ensuite, Cléanthis révèle avoir utilisé un discours pour duper sa patronne :

Cléanthis : [...] Un jour qu'elle pouvait m'entendre, et qu'elle croyait que je ne m'en doutais pas, je parlais d'elle, et je dis : Oh ! pour cela, il faut l'avouer, Madame est une des plus belles femmes du monde. Que de bontés, pendant huit jours, ce petit mot-là ne me valut-il pas !...

Le DD est introduit par le verbe je dis, définissant l'action comme ponctuelle et passée. L'énonciateurpersonnage, Cléanthis, s'adresse à Trivelin. Euphrosine assiste mais n'intervient pas. Cléanthis explique le cadre énonciatif du DD qu'elle va rapporter : elle-même en est l'énonciateur, tandis que la fonction allocutaire du discours enchâssé comporte un dédoublement intentionnel : il y a celui avec qui Cléanthis est en train de parler (qui n'est pas indiqué) et puis Euphrosine, qui est le véritable allocutaire visé, mais qui ne doit pas s'en apercevoir. L'incise il faut l'avouer insérée dans le DD souligne la sincérité de l'énonciateur en augmentant la crédibilité du propos. Remarquons dans cette autocitation la présence de l'interjection $O h$ !, uniquement possible en DD (cf. Cigada 2012a : 464 et 471).

Le récit prouve encore une fois la vanité d'Euphrosine. La littéralité qu'on imagine est fonction du statut d'épreuve, presque judiciaire, de ces DR.

\subsubsection{Exemple - Regardez mes grâces (scène 3, pp. 50)}

Finalement, Cléanthis dénonce la mise faussement négligée de sa patronne, visant en réalité à souligner la beauté sans façon de la dame et, en même temps, la simplicité de sa tenue.

Cléanthis : [...] on y montre sa bonne façon naturelle ; on y dit aux gens : Regardez mes grâces, elles sont à moi, celles-là ; et d'un autre côté on veut leur dire aussi : Voyez comme je m'habille, quelle simplicité, il n'y a point de coquetterie dans mon fait.

L'esclave s'adresse toujours à Trivelin, en présence d'Euphrosine. L'expression on y dit sert pour introduire le premier DD : c'est la dame qui parle en DD (cf. l'adjectif possessif dans mes grâces), en annonçant aux gens le message véhiculé par ses habits. Ce DD «traduit» en énonciation l'intention communicative des habits, intention que Cléanthis rend par un impératif (Regardez). L'allocutaire, les gens, est presque universel. Ce discours s'adresse en effet à tous ceux qui voient Euphrosine. 
Le deuxième DD comporte les mêmes énonciateurs, mais il est introduit par la locution vouloir dire, qui récupère ici sa nature compositionnelle en exprimant l'intention de tromper par une fausse évidence (Voyez).

\subsection{Phèdre}

Il n’y a qu'un DD dans Phèdre, les derniers mots d'Hippolyte : Théramène raconte à Thésée la fin du prince en rapportant ses paroles. Le récit commence au vers 1498, en réponse à la question de Thésée, qui s'inquiète de son fils, et se termine au vers 1570 , au cours de la scène 6 , soit l'avant-dernière de la pièce.

\subsubsection{Exemple - À ces mots, ce héros expiré (Acte V, scène 6, vv. 1561-1567)}

Théramène : [...] J'arrive, je l'appelle, et me tendant la main,/ Il ouvre un œil mourant qu'il referme soudain :/ «Le ciel, dit-il, m'arrache une innocente vie./ Prends soin après ma mort de la triste Aricie./ Cher ami, si mon père un jour désabusé/ Plaint le malheur d'un fils faussement accusé,/ Pour apaiser mon sang et mon ombre plaintive,/ Dis-lui qu'avec douceur il traite sa captive,/ Qu'il lui rende... » À ces mots, ce héros expiré,/ N'a laissé dans mes bras...

Le verbe du discours citant est dit-il, cohérent au temps présent employé par Théramène dans son récit. À remarquer que, d'après le récit de Théramène, Hippolyte parle les yeux fermés, après les avoir rouverts à son approche. Cette notation concernant le geste qui accompagne le discours est intéressante aussi : en se raccordant à la description de l'agonie, elle souligne surtout que l'action jouée sur scène à ce moment n'est faite que des paroles d'Hippolyte, le contact entre les interlocuteurs étant représenté - de manière beaucoup plus forte que par le regard - par la main tendue à l'ami ${ }^{12}$. C'est pourquoi on imagine ce discours comme rapporté de manière plutôt littérale, dans le cadre fictionnel du discours-pièce. Pour ce qui concerne le cadre énonciatif: l'énonciateur-personnage Théramène s'adresse à l'allocutairepersonnage Thésée. Le DR a été prononcé par un énonciateur, Hippolyte, qui coïncide avec un autre personnage, et adressé à Théramène. Le DD comporte par ailleurs un effet de mise en abyme parce qu'il contient la prière, adressée du fils à son père, de concéder la liberté à Aricie. Cette prière est formulée en DR indirect (dis-lui qu'avec douceur il traite sa captive, qu'il lui rende...).

Le DR d'Hippolyte demeure inachevé. C'est à travers le récit de Théramène qu'il trépasse sur scène, sa dernière «action » étant son discours: Théramène le rapporte, puis, après la pause qui marque le trépas d'Hippolyte, récupère son rôle d'énonciateur pour poursuivre la narration ( $\dot{A}$ ces mots, ce héros expiré.... $)^{13}$.

Du point de vue fonctionnel, ce DD contient le dernier témoignage de l'innocence d'Hippolyte et sa consigne à Théramène de prendre soin de son aimée. En racontant ce qu'Hippolyte a dit, Théramène exécute aussi la volonté de ce dernier de demander à Thésée sa clémence envers Aricie. Théramène est bien plus qu'un rapporteur, car il n'attend pas que Thésée soit un jour désabusé pour lui dévoiler l'innocence de son fils : le DD qu'il rapporte a justement la fonction de prouver ce qu'il a déjà affirmé en arrivant auprès de Thésée (J'ai vu des mortels mourir le plus aimable,/ Et j'ose dire encore, Seigneur, le moins coupable, vv. 1493-4). Son récit, et son récit de parole en particulier, remplit en effet une fonction argumentative, en amalgame pragmatique (récit et argumentation). Finalement, ce DD remplit la fonction d'éthopée en nous montrant jusqu'aux dernières conséquences le caractère entier d'Hippolyte, pieux envers les dieux et envers son père dont il ne veut pas dénoncer la honte, confiant dans l'amitié de Théramène, amoureux d'Aricie et inquiet de son sort, étranger à la vengeance. Par ses mots, il suggère au père une action réparatrice de tout le mal qui lui a été fait.

\subsection{Le Misanthrope}

Dans cette pièce (comme dans Cyrano), le rôle de la parole est central : en tant que franche et immuable, la parole d'Alceste distingue cet homme des autres, qui changent constamment d'avis selon les intérêts du moment. Les DD se trouvent au début de la pièce et dans l'Acte IV : dans le dernier acte c'est plutôt la 
parole écrite (la lettre de Célimène, Acte $V$, scène 4, insérée en prose après le vers 1690) qui est citée, en prenant une place importante dans le récit.

\title{
4.6.1 Exemple - Quel besoin si pressant avez-vous de rimer ? (Acte I, scène 2, 362-372)
}

Avant de répondre de manière directe à Oronte, qui s'inquiète de son jugement à propos de ses vers, Alceste essaie de lui faire comprendre son opinion en feignant de lui rapporter ce qu'il avait dit à un autre poète dans une occasion semblable.

\begin{abstract}
Oronte : Est-ce que j'écris mal ? [...]
Alceste : Je ne dis pas cela ; mais enfin lui disois-je :/ Quel besoin si pressant avezvous de rimer?/ Et qui diantre vous pousse à vous faire imprimer?/ Si l'on peut pardonner l'essor d'un mauvais livre,/ Ce n'est qu'aux malheureux qui composent pour vivre./ Croyez-moi, résistez à vos tentations,/ Dérobez au public ces occupations ;/ Et n'allez point quitter, de quoi que l'on vous somme,/ Le nom que dans la cour vous avez d'honnête homme,/ Pour prendre, de la main d'un avide imprimeur,/ Celui de ridicule et misérable auteur./ C'est ce que je tâchais de lui faire comprendre.
\end{abstract}

L'énoncé en DD est introduit par le verbe lui disois-je, renvoyant à une situation passée d'une certaine durée (une conversation). L'usage du DD permet la présence de structures syntaxiques qui ne pourraient pas apparaître en discours indirect, comme les deux énoncés interrogatifs en fonction de question (balançant entre question et question rhétorique). La deuxième question comporte aussi l'exclamation (diantre), typique du DD. Les impératifs en fonction d'incise (croyez-moi) et d'ordre (résistez ; dérobez ; n'allez point quitter) requièrent eux aussi le DD. Alceste est l'énonciateur-personnage et Oronte l'allocutaire-personnage. Pour ce qui concerne l'énoncé en DD inséré dans la réplique d'Alceste, Alceste en est l'énonciateur et «quelqu'un, dont je tairai le nom » (v. 343) l'allocutaire. Celui-ci n'a jamais la parole, sa présence demeurant bornée à celle d'allocutaire de ce discours d'Alceste.

Le DR est ici le stratagème discursif utilisé par Alceste pour permettre à son interlocuteur (Oronte) de saisir à travers le dialogisme que ce discours qui a été adressé à un autre est, maintenant, adressé à lui. Cette interprétation délie le DR du contrat de littéralité. À un niveau plus stratégique, c'est Molière qui met en scène la sottise d'Oronte, incapable de comprendre une fonction discursive si évidente. À la différence de, par exemple, 4.3.4 (C'était une Fâcheuse), ce dialogisme n'a aucune pertinence pour l'éthopée de l'allocutaire.

\subsubsection{Exemple - Si le Roi m'avoit donné/ Paris, sa grand'ville/... (Acte I, scène 2, 393- 400 et 405-412)}

Alceste cite la « vieille chanson » en la comparant au sonnet d'Oronte, qu'Oronte a déclamé peu de vers auparavant et dont Alceste vient de reprendre quelques expressions pour les commenter. Alceste répète la chanson deux fois ${ }^{14}$ :

Alceste : [...] une vieille chanson que je m'en vais vous dire :

Si le Roi m'avoit donné/ Paris, sa grand'ville,/ Et qu'il me fallût quitter/L'amour de ma mie,/ Je dirois au roi Henri :/ "Reprenez votre Paris :/ j'aime mieux ma mie, au gué !/ J'aime mieux ma mie. »...

La chanson est introduite par la forme je m'en vais vous dire: le futur proche annonce la réalisation immédiate de l'acte, mais le texte rapporté relève d'un passé éloigné, comme l'expression une vieille chanson l'indique. L'énonciateur-personnage Alceste s'adresse à l'allocutaire-personnage Oronte; la chanson en tant que DR n'est attribuée à aucun énonciateur en particulier, mais le chanteur y parle, à la première personne du singulier, sans s'adresser à aucun allocutaire en particulier. Le statut de cet exemple est ambigu, de manière analogue à 4.1.1 (Un chien vint dans l'office), même s'il y a ici la présence du verbe introducteur. Mais par contre la notion de littéralité n'a pas beaucoup de sens par rapport à ce type de citation. 
À remarquer qu'il y a un autre DD adressé par le chanteur au roi Henri, enchâssé dans la chanson avec un effet de mise en abyme. Ce DD, introduit par le conditionnel je diroit, comporte une énonciation irréelle, en fonction de paradoxe. L'énonciateur-chanteur se feuillette dans le passé.

Alceste introduit la deuxième occurrence de la chanson en invitant Oronte à percevoir la pureté de la passion qui inspire ce texte, en dépit de la pauvreté de son style. Il l'invite à l'écouter en proposant un déchiffrage lié au contexte : c'est justement la pauvreté du style (l'absence de colifichets) qui rend la passion parlante :

Alceste : La rime n'est pas riche, et le style en est vieux :/ Mais ne voyez-vous pas que cela vaut bien mieux/ Que ces colifichets, dont le bon sens murmure,/ Et que la passion parle là toute pure ?/ Si le Roi...

La répétition de la citation sert donc à préciser l'écoute : la voix, qui a été entendue (et probablement reconnue) lors de la première citation, doit ensuite être écoutée et observée selon des critères précis ${ }^{15}$. Le cadre énonciatif ne change pas, à part pour l'absence de verbe citant substitué par l'invitation à regarder l'objet-discours (ne voyez-vous pas... ?).

\title{
4.6.3 Exemple - je ne me dédis point (Acte IV, scène 1, 1139-1154 et 1158-1160)
}

Philinte raconte à Éliante le procès entre Alceste et Oronte. La scène n'a pas été jouée sur scène, c'est à travers ce récit qu'Éliante et le spectateur en connaissent l'issue :

\begin{abstract}
Philinte : [...] « Non, Messieurs, disoit-il, je ne me dédis point,/ Et tomberais d'accord de tout, hors de ce point./ De quoi s'offense-t-il ? et que veut-il me dire ?/ Y va-t-il de sa gloire à ne pas bien écrire ?/ Que lui fait mon avis, qu'il a pris de travers ?/ [...] lorsque d'en mieux faire on n'a pas le bonheur,/ On ne doit de rimer avoir aucune envie,/ Qu'on n'y soit condamné sur peine de la vie. »//Enfin toute la grâce et l'accommodement/ où c'est, avec effort, plié son sentiment,/ C'est de dire, croyant adoucir bien son style :/ « Monsieur, je suis fâché d'être si difficile,/ Et pour l'amour de vous, je voudrois, de bon cœur,/ Avoir trouvé tantôt votre sonnet meilleur. »...
\end{abstract}

Le premier verbe citant (disoit-il) à l'imparfait signale une action d'une certaine durée ou répétée, c'est-adire qu'il reproduit - sans prétension d'exactitude - l'attitude d'Alceste au procès. L'énonciateur de cet énoncé enchâssé est un personnage, Philinte, qui s'adresse à un autre personnage, Éliante, en rapportant le discours d'Alceste (personnage) qui parle aux juges (Messieurs). Ceux-ci ne sont pas par contre des personnages et n'ont jamais la parole à aucun niveau du discours-pièce. Oronte est présent, mais non pas en tant qu'allocutaire visé par ce premier DD : Alceste parle en effet de lui à la troisième personne (De quoi s'offense-t-il ?...). Les termes d'adresse (Messieurs puis Monsieur dans le deuxième DD) ne sont possibles qu'en DR direct, tout comme les quatre énoncés interrogatifs.

Le deuxième DD prévoit le verbe introducteur ...c'est de dire, accompagné par une annotation qui caractérise l'attitude d'Alceste (croyant adoucir bien son style) et manifeste celle de l'énonciateur enchâssant, Éliante. L'énonciateur en est toujours Alceste, qui s'adresse maintenant à Oronte en le vouvoyant, tandis que les juges écoutent sans prendre la parole.

Si, par le premier DD, Alceste confirme son jugement négatif des vers d'Oronte, par le deuxième, il s'excuse : tous deux collaborent à l'éthopée, en montrant la bizarrerie du tempérament excessif d'Alceste. Le maintien de la parole prononcée est le trait qui distingue Alceste de tous les autres et son refus de la changer en est une conséquence ${ }^{16}$. Ceci est perceptible en comparant ces DR aux discours mis en scène dans les répliques d'Alceste : les paroles d'Alceste rapportées ici en DD reprennent ce qu'il a déjà déclaré plusieurs fois pendant la pièce. Ces DR reprennent donc un discours antérieur, prononcé directement par le protagoniste, en constituant un dialogisme que le spectateur est censé reconnaitre. 


\title{
4.7 Le Cid
}

Dans le Cid nous avons repéré quatre cas de $\mathrm{DD}$, dont l'un se trouve dans la première scène du premier Acte, les autres dans le cinquième Acte, scène 1, 6 et 7 (la dernière).

\subsubsection{Exemple - Un si charmant discours ne se peut... (Acte I, scène 1, vv. 25-38)}

La gouvernante Elvire rapporte à Chimène le discours de don Gomès son père à propos de ses deux prétendants, la pièce s'ouvrant sur le récit de ce discours, qu'Elvire a déjà rapporté, mais que Chimène souhaite écouter à nouveau.

\begin{abstract}
Elvire : [...] Et puisqu'il vous en faut encor faire un récit,/Voici d'eux et de vous ce qu'en hâte il m'a dit :/ «Elle est dans le devoir, tous deux sont dignes d'elle,/ Tous deux formés d'un sang noble, vaillant, fidèle,/ Jeunes, mais qui font lire aisément dans leurs yeux/ L'éclatante vertu de leurs braves aïeux./ Don Rodrigue surtout n'a trait en son visage/ Qui d'un homme de cœur ne soit la haute image,/ Et sort d'une maison si féconde en guerriers/ Qu'ils y prennent naissance au milieu des lauriers./ La valeur de son père en son temps sans pareille,/ Tant qu'a duré sa force, a passé pour merveille ;/ Ses rides sur son front ont gravé ses exploits,/ Et nous disent encor ce qu'il fut autrefois./ Je me promets du fils ce que j'ai vu du père ;/ Et ma fille, en un mot, peut l'aimer et me plaire »./ Il allait au conseil..
\end{abstract}

Le DD est introduit par un verbe citant au passé proche, il m'a dit, qui indique une action ponctuelle, passée mais encore prochaine. Le discours précédent signale explicitement qu'il s'agit d'un récit et que, à l'intérieur du discours fictif de la pièce, Elvire l'a déjà répété plusieurs fois. Par ailleurs, l'énoncé cité a été prononcé en hâte. L'énonciateur et l'allocutaire de la réplique enchâssante sont respectivement Elvire et Chimène ; l'énonciateur et l'allocutaire de l'énoncé enchâssé sont don Gomès père de Chimène, personnage de la pièce ayant la parole en propre, et Elvire elle-même. Le DR porte sur Chimène et ses prétendants. En accord avec les attentes de l'allocutaire (Chimène) et avec la bonne volonté de l'énonciateur (Elvire), ce DD est censé reproduire de manière très fidèle le discours qu'il rapporte.

L'énoncé en DD manifeste une fonction narrative en constituant un élément important du récit-pièce (le spectateur apprend les relations entre plusieurs personnages principaux; Chimène apprend que son père approuve l'amour qui la lie à Rodrigue). Il manifeste aussi une fonction argumentative parce qu'il établit pour la première fois dans le discours-pièce la hiérarchie de valeurs qui en définissent le monde. De plus, pour la même raison, il est fonctionnel à l'éthopée de Rodrigue, mais aussi à celles de Chimène et de don Gomès.

\subsubsection{Exemple - II adorait Chimène... » (Acte V, scène 1, vv. 1533-1542)}

Don Rodrigue : [...] On dira seulement : « Il adorait Chimène ;/ Il n’a pas voulu vivre et mériter sa haine ;/[...] Pour venger son honneur il perdit son amour,/ Pour venger sa maîtresse il a quitté le jour,/ Préférant (quelque espoir qu'eût son âme asservie)/ Son honneur à Chimène, et Chimène à sa vie ».

La locution on dira introduit un DD placé dans l'avenir et destiné à être répété plusieurs fois : cette dernière valeur est exprimée par l'impersonnel on. L'indicatif signale que cette action discursive est certaine et l'adverbe seulement accompagnant le verbe exclut la possibilité d'autres discours concernant la mort de Rodrigue. L'énonciateur-personnage est Rodrigue même, qui s'adresse à Chimène, allocutairepersonnage. L'énonciateur et l'allocutaire de l'énoncé enchâssé n'ont pas d'identité précise, ce discours étant plutôt destiné au consensus universel. Le DD concerne les deux énonciateurs qui se l'échangent. Nonobstant sa collocation dans l'avenir, l'énonciateur garantit à son discours un haut degré de « littéralité »...

Ce DD contient l'argument fondamental que Rodrigue émet pour expliquer à Chimène qu'il se laissera tuer par Sanche, sans pourtant sacrifier son honneur et l'honneur de Chimène. L'honneur a en effet dans cette pièce une dimension exquisément sociale : c'est devant les autres qu'il doit être préservé. L'effet 
que Rodrigue cherche est de reproduire les discours que sa mort causera, ce que l'on en dira, pour vérifier et montrer à Chimène que ces discours rehausseront - au lieu d'amoindrir - l'honneur de chacun d'eux. La démonstration force Chimène, dans la réplique suivante, à changer d'attitude : elle demande en effet à Rodrigue de vaincre, pour l'affranchir de Sanche.

\subsubsection{Exemple - Ne crains rien (Acte V, scène 6, vv. 1747-1753)}

Dans le récit du duel, don Sanche rapporte en DD les mots que Rodrigue lui a adressés après l'avoir désarmé.

Don Sanche : [...] Ce généreux guerrier, dont son cœur est charmé,/ « Ne crains rien, » m'a-t-il dit, quand il m'a désarmé :/ « Je laisserais plutôt la victoire incertaine,/ Que de répandre un sang hasardé pour Chimène;/ Mais puisque mon devoir m'appelle auprès du roi,/ Va de notre combat l'entretenir pour moi,/ De la part du vainqueur lui porter ton épée »./ Sire,...

Le verbe qui introduit l'énoncé enchâssé est dire ( $m^{\prime}$ 'a-t-il dit), relatant en évènement ponctuel, passé mais récent. L'énonciateur-personnage don Sanche parle devant la cour, en présence de Chimène, en s'adressant directement au roi qu'il appelle Sire. Il rapporte dans l'énoncé en DD le discours tenu par ce généreux guerrier (Rodrigue, énonciateur-personnage) à lui-même. Rodrigue n'est pas présent et le DR n'a pas été joué sur scène.

Ce DD couronne l'éthopée de Rodrigue : il est encore une fois fidèle à la hiérarchie de ses devoirs, il obéit à Chimène en vainquant Sanche et son amour justifie qu'il l'épargne, mais l'amour ne l'empêche pas de courir avant tout chez le roi.

\subsubsection{Exemple - S'il ne m'avait aimée, il ne serait pas mort (Acte $V$, scène 7, v. 1800)}

Le dernier DD de la pièce contient les mots que Rodrigue demande à Chimène de répéter en son souvenir :

Don Rodrigue : [...] Et dites quelquefois, en déplorant mon sort :/ «S'il ne m'avait aimée, il ne serait pas mort ».

La requête est formulée par le verbe citant à l'impératif dites : elle concerne un avenir hypothétique. L'énonciateur et l'allocutaire-personnage en sont respectivement Rodrigue et Chimène, tandis que, pour l'énoncé enchâssé, l'énonciateur en est Chimène s'adressant à soi-même. Le cadre énonciatif rappelle donc 4.1.2 (Pense, cochon!) mais n'est pas identique.

L'avenir dont on parle dépendra du sentiment de Chimène, de son choix entre l'amour de Rodrigue ou la mort de celui-ci (car en tout cas il ne veut plus survivre sans elle). Et si elle décrétait sa mort, il suggère à Chimène de se répéter souvent que seul l'amour a causé sa fin. Cette suggestion fortement émotive adressée à Chimène déclenche à nouveau la réponse de son cœur : elle accepte l'amour de Rodrigue.

\section{$5 \quad$ Résultats et conclusions préliminaires}

Nous allons résumer les résultats en suivant les premiers trois niveaux de la grille proposée au début (§ 3 ). Nous ne considérerons pas le quatrième niveau: il suffit, à cette étape de notre sondage, d'avoir vérifié que les DD peuvent remplir les fonctions, très variées, résultant de l'analyse des exemples.

1. Fréquence des DD et leur distribution

\begin{tabular}{|ll|l|l|}
\hline & & Structure de la pièce & Distribution des DD \\
\hline 4.1 & Godot & 2 actes & Acte II (4) \\
\hline 4.2 & Topaze & 4 actes & un peu partout surtout aux Actes III (3) et IV (1) \\
\hline 4.3 Cyrano & 5 actes & Nombreux et distribués partout \\
\hline
\end{tabular}




\begin{tabular}{|ll|l|l|}
\hline 4.4 & l'île des esclaves & 11 scènes & Que dans la scène 3, une dizaine \\
\hline 4.5 & Phèdre & 5 actes & Acte V, scène 6 (avant-dernière scène), un seul \\
\hline 4.6 & le Misanthrope & 5 actes & Acte I et IV (+Acte V, la lettre) \\
\hline 4.7 le Cid & 5 actes & Acte I scène $1 ;$ Acte V scène $1,6,7$ \\
\hline
\end{tabular}

La distribution n'est vraisemblablement pas fortuite : les DD sont distribués de manière cohérente à des moments décisifs pour le déroulement du récit.

\section{Aspects morphosyntaxiques}

\begin{tabular}{|c|c|c|c|c|}
\hline & Verbes citants & Dire & $\mathrm{G}$ & $\mathrm{V}$ \\
\hline 4.1 Godot & $\begin{array}{l}\text { 4.1.1- } \\
4.1 .2 \text { dis-moi } \\
4.1 .3- \\
4.1 .4 \text { en disant }\end{array}$ & & $*$ & \\
\hline 4.2 Topaze & $\begin{array}{l}\text { 4.2.1 ils disaient ; chuchotaient } \\
\text { 4.2.2 - } \\
\text { 4.2.3 (il) disait ; vous avez répondu } \\
\text { 4.2.4 je dirais }\end{array}$ & * & $*$ & \\
\hline 4.3 Cyrano & $\begin{array}{l}\text { 4.3.1 on pouvait dire ; vous m'auriez dit } \\
\text { 4.3.2 je disais } \\
\text { 4.3.3 je répondais ; en disant ; j'ai dit ; si j'avais dit } \\
\text { 4.3.4 j'ai dit }\end{array}$ & $*$ & $\begin{array}{l}* \\
*\end{array}$ & $*$ \\
\hline 4.4 l'île des esclaves & $\begin{array}{l}\text { 4.4.1 (vous allez voir) ; et cela veut dire } \\
\text { 4.4.2 disiez-vous; lui dîtes-vous; - ; dîtes-vous } \\
\text { 4.4.3 je dis (passé) } \\
\text { 4.4.4 on y dit ; on veut leur dire }\end{array}$ & $\circ$ & $* *$ & \\
\hline 4.5 Phèdre & 4.5.1 dit-il (prés.) & & $*$ & \\
\hline 4.6 le Misanthrope & $\begin{array}{l}\text { 4.6.1 lui disois-je } \\
\text { 4.6.2 je m'en vais vous dire; (ne voyez-vous pas ?) } \\
\text { 4.6.3 disoit-il ; c'est de dire }\end{array}$ & $\circ$ & $\circ$ & \\
\hline 4.7 le Cid & $\begin{array}{l}\text { 4.7.1 il m'a dit } \\
\text { 4.7.2 on dira } \\
\text { 4.7.3 m'a-t-il dit } \\
\text { 4.7.4 dites (imp.) }\end{array}$ & & $*$ & \\
\hline
\end{tabular}

Nous avons indiqué dans la colonne Bire l'occurrence de verbes différents de dire, qui se réduisent à deux occurrences de répondre (4.2.3 Topaze et 4.3.3 Cyrano), à chuchoter (4.2.1, dans Topaze) et à deux occurrences de voir (4.4.1 et 4.6.2). L'absence de verbe citant coïncide avec les cas d'autonymie sauf pour 4.4.2 (troisième DD au cours du dialogue entre Euphrosine et le cavalier). Dans la colonne G nous avons indiqué la présence de marques concernant l'action ou les gestes qui accompagnent le dire : parmi les gestes, nous avons noté aussi 4.7.1 (en hâte); 4.6 .3 et 4.7.4 signalent plutôt une attitude psychologique attribuée à l'énonciateur (croyant adoucir bien son style et en déplorant mon sort). Les gestes accompagnent donc assez régulièrement la parole, ce qui semble plutôt naturel si l'on considère que le récit comporte l'action verbale mêlée dans l'action. À remarquer qu'aucun cas comportant un geste, sauf pour 4.3.2, ne corresponde à une autocitation : l'énonciateur-personnage décrit donc « l'action et le discours » d'un autre. La présence de marques qui décrivent la voix ou la manière de parler se trouve dans la colonne V: il n'y a que dans 4.3.2 que le verbe citant est connoté du point de vue métaphonologique (je disais d'une voix qui cherchait d'être dure).

Ces données sont probablement à imputer à la situation discursive : d'un côté au besoin de baisser le niveau de difficulté d'interprétation. De l'autre, le texte étant écrit pour être dit, les effets prosodiques n'ont pas besoin d'être décrits : l'auteur peut laisser à l'acteur le soin de donner au DD la voix la plus adaptée. Ce résultat est significatif par rapport au statut de la voix physique (l'acteur) qui joue le 
personnage : on ne peut pas effacer tout court cette médiation car elle a apparemment une influence sur l'organisation du cadrage énonciatif.

À propos des termes d'adresse, on remarque une évolution du registre, du plus formel au moins formel ; les interjections (en italique) n'apparaissent que dans les pièces les plus anciennes; les énoncés interrogatifs, impératifs et exclamatifs semblent être distribués de manière plutôt uniforme, comme le tableau ci-dessous le montre, mais il serait sans aucun doute convenable de les étudier davantage.

\begin{tabular}{|l|l|l|}
\hline & Termes d'adresse & Syntaxe de l'énoncé en DD \\
& interjections & \\
\hline Godot & 4.1 .1 & déclaratif (un chien vint) \\
& 4.1 .2 cochon & impératif (Pense !) \\
& 4.1 .3 & déclaratif tronqué \\
& 4.1 .4 & déclaratif (il dort...) ; subjonctif (qu'il dorme) \\
\hline Topaze & 4.2 .1 & exclamation sans verbe (tripoteur ! Topaze escroc !) \\
& 4.2 .2 & exclamatif (cachât !) \\
& 4.2 .3 chérie & interrogatif (pourquoi... ?) ; déclaratif (il est très utile) \\
& 4.2 .4 mes enfants & déclaratif (les proverbes correspondaient...) \\
\hline Cyrano & 4.3 .1 monsieur & exclamatif (...) \\
& 4.3 .2 & interrogatif (qu'est-ce que c'est... ?) \\
& 4.3 .3 señorita & déclaratif (je vais); impératif (passez) \\
& 4.3 .4 & impératif (excusez-moi ; repassez !) \\
\hline 'Île & 4.4 .1 Madame, Messieurs & interrogatif; déclaratif ; impératif \\
des esclaves & 4.4 .2 Madame, folâtre & déclaratif; interrogatif (à moi ?); exclamatif (à vous !..) \\
& 4.4 .3 Oh! & déclaratif \\
& 4.4 .4 & impératif (regardez mes grâces ! voyez... !) \\
\hline Phèdre & 4.5 .1 cher ami & déclaratif; impératif (prends soin ; dis-lui que) \\
\hline le Misanthrope & 4.6 .1 & interrogatif rhét.; déclaratif ; exclamatif (croyez-moi...) \\
& 4.6 .2 au gué ! & déclaratif conditionnel \\
& 4.6 .3 Messieurs, Monsieur & interrogatif; déclaratif; déclaratif \\
\hline le Cid & 4.7 .1 & déclaratif (elle est dans le devoir) \\
& 4.7 .2 & déclaratif (il adorait Chimène) \\
& 4.7 .3 & exclamatif (ne crains rien) ; déclaratif ; exclamatif \\
& 4.7 .4 & déclaratif conditionnel (...il ne serait pas mort) \\
\hline
\end{tabular}

3. Ces signaux permettent d'étudier le cadre énonciatif. L'analyse des verbes introducteurs rend possible en effet de distinguer les cas d'autocitation (colonne AC) des autres cas, que nous avons classés en énoncés attribués à l'allocutaire du discours citant (colonne All); énoncés attribués à un autre personnage (colonne AutreP) ; énoncés attribués à un énonciateur qui n'est pas un personnage (colonne autre).

On constate que la distribution des phénomènes observés change d'un ouvrage à l'autre. L'autocitation ne concerne pas Godot, Phèdre et le Cid. La citation d'autres énonciateurs externes au discours concerne plutôt les pièces plus récentes. Il faudra étudier plus de données, parce que la distribution paraît être significative.

Par rapport au feuilletage des énonciateurs, on remarque la possibilité d'un « auto-feuilletage », qui peut être conditionnel (comme dans 4.2.4, je dirais), passé ponctuel mais habituel (4.3.2 je disais; 4.3 .3 je répondais), passé ponctuel (4.3.3 j'ai dit; 4.3 .4 j'ai dit; 4.4 .3 je dis), irréel (4.3.3 si j'avais dit), passé prolongé (4.6.1 lui disais-je), futur proche (4.6.2 je m'en vais vous dire). D'autre part, le feuilletage peut concerner aussi l'allocutaire-énonciateur (colonne All), dont le discours peut être convoqué par l'énonciateur à travers un impératif qui concerne l'avenir (4.1.2 dis-moi; 4.7 .4 dites), ou par une déclarative qui concerne le passé (4.2.3 vous avez répondu; 4.4.2 disiez-vous, lui dîtes-vous et dîtes-vous) 
mais aussi le présent (4.4.4 on y dit et on veut leur dire), comme une possibilité irréelle (4.3.1 on pouvait dire et vous m'auriez dit). Plusieurs cas de figure apparaissent donc dans notre exemplier : aux remarques de Rosier sur le "'sujet' se feuilletant en des 'mois' passés » (Rosier 2008 : 126-132), on peut ajouter que, outre la possibilité de «re-convoquer son propre dit» (127), le feuilletage peut convoquer aussi les 'mois' que l'on vient de considérer.

Par ailleurs, les discours des autres personnages (AutreP) se situent toujours dans le passé. En revanche, les discours attribués à des énonciateurs externes à la pièce (autre) peuvent se situer dans le passé (4.1.1 un passant pouvait lire; 4.1.3 Cicéron; 4.2.1 les balayeuses disaient et chuchotaient ; 4.3 .3 l'Espagnol s'inclinait en disant), mais aussi dans le présent (4.1.4 un autre me regarde en se disant) et dans l'avenir (4.7.2 on dira).

Parmi les formes impersonnelles (on) qu'on retrouve en 4.3.1, 4.4.4 (deux fois) et 4.7.2, les trois premières renvoient en réalité à l'allocutaire correspondant en effet respectivement à vous pouviez dire et vous y dites. Le troisième représente par contre un véritable impersonnel, comme on l'a dit au cours de l'analyse.

\begin{tabular}{|c|c|c|c|c|c|}
\hline & Verbes citants & $\mathrm{AC}$ & All & AutreP & autre \\
\hline 4.1 Godot & $\begin{array}{l}4.1 .1- \\
4.1 .2 \text { dis-moi } \\
4.1 .3- \\
4.1 .4 \text { en se disant } \\
\end{array}$ & & * & & \\
\hline 4.2 Topaze & $\begin{array}{l}\text { 4.2.1 ils disaient; chuchotaient } \\
4.2 .2- \\
\text { 4.2.3 (il) disait ; vous avez répondu } \\
\text { 4.2.4 je dirais }\end{array}$ & * & & * & $* *$ \\
\hline 4.3 Cyrano & $\begin{array}{l}\text { 4.3.1 on pouvait dire ; vous m'auriez dit } \\
4.3 .2 \text { je disais } \\
\text { 4.3.3 je répondais ; en disant ; j'ai dit ; si j'avais dit } \\
\text { 4.3.4 j'ai dit }\end{array}$ & $\begin{array}{l}* \\
* * * \\
*\end{array}$ & ** & & * \\
\hline $\begin{array}{l}4.4 \text { l'Île } \\
\text { des esclaves }\end{array}$ & $\begin{array}{l}\text { 4.4.1 (vous allez voir) ; et cela veut dire } \\
\text { 4.4.2 disiez-vous; lui dîtes-vous; - ; dîtes-vous (passé) } \\
\text { 4.4.3 je dis (passé) } \\
\text { 4.4.4 on y dit ; on veut leur dire }\end{array}$ & * & $* * *$ & & $?$ \\
\hline 4.5 Phèdre & 4.5.1 dit-il (prés.) & & & $*$ & \\
\hline $\begin{array}{l}4.6 \\
\text { Misanthrope }\end{array}$ & $\begin{array}{l}\text { 4.6.1 lui disois-je } \\
\text { 4.6.2 je m'en vais vous dire; (ne voyez-vous pas ?) } \\
\text { 4.6.3 disoit-il ; c'est de dire }\end{array}$ & * & & $* *$ & \\
\hline 4.7 le Cid & $\begin{array}{l}\text { 4.7.1 il m'a dit } \\
\text { 4.7.2 on dira } \\
\text { 4.7.3 m'a-t-il dit } \\
\text { 4.7.4 dites (imp.) }\end{array}$ & & $*$ & * & * \\
\hline
\end{tabular}

Pour ce qui que concerne le cadre énonciatif, il ne nous reste à considérer que la position de l'allocutaire du DD, au sein de la pièce. Les cas de figure sont nombreux, parce que le DR peut s'adresser :

- à l'énonciateur, qui rapporte le discours d'un autre (4.3.3 en [me] disant ; 4.4.1. et 4.4.2 présentent des situations de double allocutaire du fait que Cléanthis à assisté aux conversations entre la dame et d'autres personnes ; 4.5.1 [me] dit-il ; 4.7.1 il m'a dit ; 4.7.3 m'a-t-il dit) ;

- à l'énonciateur, en échangeant le rôle des deux (comme dans Godot 4.1.2 dis-moi; Topaze 4.2.2 cachât et Cyrano 4.3.1 on pouvait dire et vous m'auriez dit) ;

- à l'allocutaire du discours citant (Topaze 4.2.3 il [vous] disait; île des esclaves 4.4.2 le DD du cavalier, sans verbe introducteur); en cas d'autocitation, le DD reproduit alors le cadre citant (Cyrano 4.3.2 je [vous] disais ; Misanthrope 4.6.2 je m'en vais vous dire);

- à un autre des personnages (Topaze 4.2.3 vous [lui] avez répondu); 
- à un allocutaire externe au discours-pièce, particulier (4.1.4 un autre disant à soi-même; 4.3.3 je [à l'Espagnol] répondais ; j'ai dit; si j'avais dit ; 4.3 .4 j'ai dit [à la Fâcheuse] ; 4.6 .1 lui disois-je [à quelqu'un dont je tairai le nom] ; 4.6 .3 disoit-il [aux juges]) ou universel (4.1.1 ; 4.1.3 ; 4.2.1 ; 4.7.4 où l'allocutaire pourrait aussi être à vous-même).

Quand les énonciateurs rapportés n'apparaissent pas dans la pièce comme personnages, le DD fait rentrer leurs discours dans le discours dit en scène, de manière totalement exogène. À remarquer que, parfois, ces «énonciateurs » ne participent du cadre énonciatif qu'en tant qu'allocutaires du DR, sans jamais prendre la parole. En récapitulant, on observe que les cadres énonciatifs constitués par les répliques d'une pièce de théâtre peuvent enchâsser des DD selon plusieurs types de cadres énonciatifs subordonnés. Nous soulignons en particulier la possibilité d'inclure dans le discours-pièce, par le biais du DR, des énoncés qui mettent en rapport le discours-pièce et ses énonciateurs avec des discours prononcés par ou adressés à d'autres énonciateurs/allocutaires qui ne font pas partie du cadre citant.

$$
* * *
$$

Ces premières conclusions confirment l'intérêt de la démarche proposée, tout en indiquant la nécessité absolue d'élargir systématiquement le corpus, avant qu'on ne puisse préciser le projet de recherche. En effet, si ce sondage ne nous permet pas de confirmer l'hypothèse émise à propos d'une évolution historique du cadre énonciatif du DD dans le discours théâtral, il permet toutefois d'apprécier des variations très fortes de celui-ci en suggérant d'approfondir la recherche, surtout en ce qui concerne 1.l'exploitation du cadre énonciatif, cf. les pièces de Rostand, Pagnol et Beckett (énonciateurs qui ne sont pas des personnages et recadrages énonciatifs conséquents) ; 2.les ouvrages où la parole est au cœur du message (cf. Molière et Rostand) ; 3.la quantité de DD utilisé et sa collocation dans la pièce (cf. Corneille, Racine et Marivaux).

\section{Références bibliographiques}

Adam, J.-M. (1992). Les Textes : types et prototypes. Paris : Nathan.

Authier-Revuz, J. (2003). Le Fait autonymique : langage, langue, discours Quelques repères. In : Parler des mots. Le fait autonymique en discours, Authier-Revuz, J., Doury, M., Reboul-Touré, S. (éds.). Paris : Presses Sorbonne Nouvelle, 67-96.

Bres, J. (2005). Savoir de quoi on parle : dialogue, dialogal, dialogique; dialogisme, polyphonie... In : Dialogisme et polyphonie, Bres, J. et al. (éds.). Bruxelles : de boeck.duculot, 47-61.

Bres, J. \& Mellet, S. (2009). Une Approche dialogique des faits grammaticaux. Langue française, 163, 3-20.

Calaseru, E. (2004). Testuali parole. Milano : F. Angeli.

Cigada, S. (2005). L'Expression des émotions dans les dialogues de 'La Princesse de Clèves'. Phénomènes d'exploitation de la coordination. In : Dialogue in Literature and the Media, Betten, A. \& Dannerer, M. (éds.). Tübingen : Niemeyer, vol. 1 Literature, 209-217.

Cigada, S. (2012a). Sur la plurifonctionnalité du discours direct. In : $3^{e}$ Congrès Mondial de Linguistique Française, Neveu, F. et al. (éds.). SHS Web of Conferences, vol. 1, 459-476.

Cigada, S. (2012b). Remarques sur la fonction du discours direct dans la narration. In: Modernitate şi interdisciplinaritate în cercetarea lingvistică. Omagiu doamnei profesor Liliana Ionescu-Ruxăndoiu [Modernité et interdisciplinarité dans la recherche linguistique. Hommage au professeur Liliana Ionescu-Ruxăndoiu], Constantinescu, M.N., Stoica, G., Bărbulescu, O.U. (éds.). Bucarest : Editura Universităţii din Bucureşti, 143160.

Combettes, B. (2012). Linguistique textuelle et diachronie. In : $3^{e}$ Congrès Mondial de Linguistique Française, SHS Web of Conferences, 1, 13-10.

Ducrot, O. et al. (1980). Les Mots du discours. Paris : Éditions de minuit. 
Dufiet, J.-P. (2005). Le Discours rapporté direct dans la comédie classique (Le cas de Molière). In : Dans la jungle des discours: genres de discours et discours rapportés, Lopez, J.M., Marnette, S., Rosier, L. (éds.). Cádiz: Servicio de publicaciones de la Universidad de Cádiz, 183-192.

Dufiet, J.-P. \& Petitjean, A. (2013). Approches linguistiques des textes dramatiques. Paris : Classiques Garnier.

Granier, J.-M. (2003). Faire référence à la parole de l'autre : quelques questions sur l'enchaînement «sur le mot » chez Marivaux. In : Parler des mots. Le fait autonymique en discours, Authier-Revuz, J., Doury, M., ReboulTouré, S. (éds.). Paris : Presses Sorbonne Nouvelle, 217-231.

Lopez Muños, J.M., Marnette, S., Rosier, L. (2004). Le Discours rapporté dans tous ses états. Paris : Harmattan.

Nowakowska, A. (2005). Dialogisme, polyphonie : des textes russes de M. Bakhtine à la linguistique contemporaine. In : Dialogisme et polyphonie, Bres, J. et al. (éds.). Bruxelles : de boeck.duculot, 19-32.

Petitjean, A. (2005). Textualité dramatique et discours rapporté : l'exemple de Marivaux. In : Dans la jungle des discours : genres de discours et discours rapportés, Lopez, J.M., Marnette, S., Rosier, L. (éds.). Cádiz : Servicio de publicaciones de la Universidad de Cádiz, 193-202.

Rigotti, E. \& Rocci, A. (2006). Le Signe linguistique comme structure intermédiaire. In : Nouveaux Regards sur Saussure. Mélanges offerts à René Amacker, Saussure, L. (éd.). Genève : Droz, 219-247.

Rosier, L. (2008). Le Discours rapporté en français. Paris : Ophrys.

Salvan, G. (2005). Dites-vous ou le dialogique à l'épreuve du dialogal (et vice-versa). In : Dialogisme et polyphonie, Bres, J. et al. (éds.). Bruxelles : de boeck.duculot, 265-279.

Sebellin, R. (2003). Quattro cani in cucina : La filastrocca in Waiting for Godot di Samuel Beckett, Linguae \& 2003/2, 41-58.

Siouffi, R. (2013). Mensonges hérö̈ques : autour de la fonction défensive de la parole chez Corneille. In : Héros ou personnages? Dufour-Maître, M. (éd.). Mont-Saint-Aignan : PURH, 129-145.

Stati, S. (1991). Le Transphrastique. Paris : PUF.

Todorov, Tz. (1981). Mikhaïl Bakhtine. Le principe dialogique. Paris : Éditions du Sueil.

\section{Sources des exemples (éditions utilisées)}

Beckett, S. (1952). En attendant Godot. Paris : Les Éditions de minuit (pour les exemples : 4.1.1-4).

Corneille, P (2012). Le Cid. Milan : Feltrinelli. Lunari, L. éd. (pour les exemples : 4.7.1-4).

Marivaux (1999). L'Île des esclaves. Paris : Le livre de poche (pour les exemples : 4.4.1-4),

Molière (1982). Le Misanthrope. Milan : BUR. Lunari, L. éd. (pour les exemples : 4.6.1-3).

Pagnol, M (2004). Topaze. Paris : Éditions de Fallois (pour les exemples : 4.2.1-4).

Racine, J (2011). Phèdre. Paris : Le livre de poche (pour l'exemple : 4.5.1).

Rostand, E. (1983). Cyrano de Bergerac. Paris : Gallimard (pour les exemples : 4.3.1-4).

\footnotetext{
${ }^{1}$ Le statut de l'acteur est par ailleurs un peu plus complexe car, si la pièce est mise en scène (et non pas lue), il prête physiquement sa voix au personnage, mais aussi aux énonciateurs que le personnage cite en DD : dans ce cas-là, l'acteur joue donc «à la frontière » entre l'énoncé enchâssé et l'énoncé enchâssant.

${ }^{2}$ L'ouvrage de Dufiet - Petitjean (2013) qui vient de paraître sera sans doute décisif pour ce sujet. La table des matières ne semble en tout cas pas indiquer une approche historique du sujet.

${ }^{3}$ Temps et modes du discours citant et, par conséquent, rapports entre le temps et la modalité du DD et le temps et la modalité de l'acte «originaire» (Rosier 2008 : 118 parle d'anticipation du discours d'autrui par pseudo-discours rapportés introduits par un verbe au futur).

${ }^{4}$ Pour renvoyer aux textes, nous avons utilisé les éditions citées en bibliographie, en indiquant les pages ou les vers, selon les cas.
} 
${ }^{5}$ Cf. Sebellin 2003 qui renvoie à J. Strauss I, Erinnerung an Ernst oder Der Carnaval in Venedig, Fantasie Op. 126. Mélodie de la comptine allemande Ein Hund kam in die Küche (mais aussi Mein Hut der hat drei Ecken). La chansonnette de Vladimir contient un discours direct écrit rapporté à l'oral qui se répète à l'infini. Cf. http://www.youtube.com/watch?v=HowoftxyPWs.

${ }^{6}$ Effet semblable mais beaucoup moins fort quand Vladimir chante « Do do do do... » pour faire dormir Estragon (Acte II, p. 99).

${ }^{7}$ Phénomène partiellement analogue dans ce discours, cité en DD mais jamais prononcé : Roxane : [...] J'ai songé : s'il voulait, lui que tous ils craindront... (Acte II, scène 6, p. 126). A propos de ce type de DD, cf. Cigada (2012b : 155, corpus Bosco Gutierrez).

${ }^{8}$ Les deux occurrences du DD dans la première partie de l'ouvrage sont utilisées par le directeur M. Muche, l'une (2004 : 21) pour citer le règlement de la pension Muche (acte I, scène 3), l'autre (2004:28) pour communiquer à Topaze qu'il n'a pas reçu les Palmes académiques (acte I, scène 3), avec un effet ironique qui sert pour souligner sa niaiserie : Muche : Il m’a dit «M. Topaze mérite dix fois les Palmes !»/ Topaze : Dix fois ! / M. : « Mérite dix fois les Palmes, et j'ai eu presque honte quand j'ai appris qu'il ne les avait pas encore. » / T. (il rougit de joie) : Oh ! je suis confus, monsieur le directeur ! / M. : «D'autant plus, a-t-il ajouté, que je ne puis pas les lui donner cette année !»/ T. (consterné) : Ah ! Il ne peut pas ! / M. : Hé, non. Il a dû distribuer tous les rubans dont il disposait à des maîtres plus anciens que vous... Tenez, reprenez votre dossier. Ses dernières paroles ont été : «Dites bien à M. Topaze que pour cette année je lui décerne les Palmes moralement. » / T. Moralement ? / M. Moralement. C'est peutêtre encore plus beau.

${ }^{9}$ La version cinématographique de 1950, réalisée par Pagnol avec Fernandel, ne conserve malheureusement pas cette réplique de Topaze. Elle est par contre gardée dans la première version, de 1936 (Pagnol, avec Arnaudy dans le rôle de Topaze). Arnaudy y exprime de manière marquée l'effet onomatopéique des deux DD reproduisant le bruit des balayeuses.

${ }^{10}$ Nous ne considérons pas, par exemple, les scènes 1 à 4 du premier acte qui se passent au théâtre et dans lesquelles se déroule le début d'une représentation précieuse, avec un effet de mise en abyme. Nous ne considérons pas non plus la ballade qui accompagne le duel (dans cette même scène 4) et que l'on pourrait classer comme DD en tant qu'autocitation ou auto-récitation (Cyrano joue son personnage de spadassin et poète, en l'affichant avec ostentation). Quelques vers de la ballade seront repris par Ragueneau (Acte II, scène 3, p. 107) qui les cite à Cyrano pour le complimenter. Un autre type encore (d'autocitation en DD) concerne l'écriture de lettres sur scène (Acte II, scène 3, p. 109), ou la déclamation de la « recette en vers » de Ragueneau (Acte II, scène 4, p. 113-4). Fonctions de mise en abyme dans la fiction de la «gazette » de Cyrano, p. 302.

${ }^{11}$ La longue liste de choses que l'on aurait pu dire contient aussi deux fonctions autonymiques introduites par le verbe appeler, dont la première est la fausse citation d'Aristophane (l'animal qu'Aristophane appelle Hippocampelephantocamélos), reprise des Lettres de Le Bret : appeler a ici la fonction de récupérer parmi les objets connus celui qui correspond au nom utilisé. La deuxième fois, appeler est utilisé pour introduire l'expression avoir pignon sur rue dans

Cyrano : [...] Respectueux : «Souffrez, monsieur, qu'on vous salue/ C'est là ce qui s'appelle avoir pignon sur rue !»

L'usage autonymique de l'expression figée n'est pas souligné du point de vue typographique, mais seulement par la présence du discours citant. Le contexte déclenche la réinterprétation de l'expression figée. Finalement Rostand introduit la dernière variation par une mise en abyme qui renvoie à Théophile de Viau, auteur de Pyrame et Thisbé : [...] Enfin parodiant Pyrame en un sanglot :/ "Le voilà donc ce nez... » (Rostand 1983 : 352, note 29) : ce DD a donc la fonction de 'pasticher' le texte de De Viau.

${ }^{12}$ Il faudrait peut-être étudier systématiquement le rapport entre regard et parole dans les pièces de théâtre en utilisant les travaux de Lorenza Mondada.

${ }^{13}$ Nous avons retrouvé sur internet un enregistrement de la Société des Comédiens Français, réalisation de J. Reynier, radiodiffusé le 16/07/1953 http://www.youtube.com/watch?v=b1zvkJwdOxY (13'05'’-30') : la récitation du DD, plutôt rapide, souligne la souffrance du mourant. À comparer à la mise en scène de P. Chéreau, Odéon-Théâtre de l'Europe, Paris 2003, http://www.youtube.com/watch?v=W lpICaJkG8 (2h8'20''-9'12') : la récitation du DD est beaucoup plus lente et inexpressive du point de vue prosodique, ce qui souligne la littéralité du DR (de plus, l'acteur ferme ses yeux, comme s'il était en train de se rappeler exactement les mots). 
${ }^{14}$ Selon la tradition interprétative, cette chanson serait chantée la première fois et dite la deuxième (Molière 1982 : 91, note 6). En fait nous avons pu consulter deux versions enregistrées : la chanson du Roi Henri est dite deux fois dans la version jouée en 2000 à la Comédie Française (mise en scène de J.-P. Miquel, Alceste joué par D. Podalydès, http://www.youtube.com/watch? $\mathrm{v}=\mathrm{ImlUzaTaM} 7 \mathrm{Q}$, minute $\left.28: 15^{\prime}\right)$, mais chantée deux fois dans la mise en scène amateur de 2011 par l'Atelier théâtre de l'École alsacienne (http://www.youtube.com/watch?v=Z57hVx624J4, minute $\left.20: 45^{\prime}\right)$.

${ }^{15}$ La répétition peut revêtir une autre fonction, de reprise en écho, quand par exemple la prude Arsinoé rend visite à Célimène pour lui rapporter les médisances qui la concernent, afin qu'elle se corrige. Célimène la remercie en lui rendant le même service. En conclusion de sa tirade, Célimène reprend exactement les derniers mots de la tirade d'Arsinoé, en la parodiant (Molière, Le Misanthrope, Acte III, scène 4, 909-912 et 957-960) :

Arsinoé : [...] Madame, je vous crois l'âme trop raisonnable,/ Pour ne pas prendre bien cet avis profitable,/ Et pour l'attribuer qu'aux mouvements secrets/ D'un zèle qui m'attache à tous vos intérêts.

Célimène : [...] Madame, je vous crois aussi trop raisonnable,/ Pour ne pas prendre bien cet avis profitable,/ Et pour l'attribuer qu'aux mouvements secrets/ D'un zèle qui m'attache à tous vos intérêts.

${ }^{16}$ Nous ne considérons pas ici le billet dont il est question dans l'Acte IV, scène 3, dont l'interprétation serait susceptible de changer totalement selon qu'il s'adresse à un homme ou à une femme (mais dont le texte n'est pas cité). Nous ne considérons pas non plus toute la scène 4 du cinquième Acte, qui tourne autour de la lecture des billets de Célimène à Clitandre et Acaste, en présence de ses amoureux, et dans lesquels elle se plaint de chacun d'eux en en décrivant les défauts. Le DD manifeste également ici le dialogisme par l'usage de la prose, insérée parmi les vers de la pièce. 